\title{
Statistics of surface renewal of passive scalars in free-surface turbulence
}

\author{
ALIREZA KERMANI ${ }^{1}$, HAMID R. KHAKPOUR ${ }^{1}$, \\ LIAN SHEN ${ }^{1,2}$ IND TAKERU IGUSA ${ }^{1}$ \\ ${ }^{1}$ Department of Civil Engineering, Johns Hopkins University, Baltimore, MD 21218, USA \\ ${ }^{2}$ Center for Environmental and Applied Fluid Mechanics, Johns Hopkins University, \\ Baltimore, MD 21218, USA
}

(Received 28 February 2010; revised 28 February 2011; accepted 6 March 2011; first published online 3 May 2011)

We perform direct numerical simulation to study the transport of gas and heat as passive scalars in free-surface turbulence. Our analysis focuses on the surface age of surface fluid particles, i.e. the time elapsed since the last surface renewal they experienced. Using Lagrangian tracing of fluid particles combined with heat diffusion analysis, we are able to directly quantify surface age to illustrate scalar characteristics at different stages of interfacial transfer. Results show that at the early stage of surface renewal, vertical advection associated with upwellings greatly enhances surface gas flux; random surface renewal model does not apply at this stage when most of the interfacial gas transfer occurs. After a fluid particle leaves the upwelling region, it may enter a nearby downwelling region immediately, where the gas flux is sharply reduced but the variation in surface temperature is small; alternatively, the fluid particle may travel along the surface for some time before it is absorbed by a downwelling, where the surface temperature has changed significantly due to long duration of diffusion and the gas flux is also reduced. To gain further insight into the relationships between surface velocity and scalar quantities, we perform a statistical analysis of upwellings using clustering and nonlinear regression. With this analysis, we are able to provide qualitative and quantitative descriptions of the skewed probability density functions associated with the surface divergence, temperature and gas flux that support our physics-based investigation of surface renewal and surface age.

Key words: channel flow, turbulent mixing

\section{Introduction}

Turbulent transport of gas and heat at an air-water interface is important to many applications including industrial equipment involving multi-phase flows, ecosystems of lakes and rivers, as well as air-sea heat and greenhouse gas transfer. To model interfacial transfer accurately, it is critical to understand the hydrodynamics of turbulent flows near the free surface. Many gases have low solubility in water and/or lower molecular diffusivity in water than in air. As a result, the rate of interfacial gas transfer is controlled by water-side fluid motion (as shown in the analyses in Liss \& Slater 1974; Jähne \& Haußecker 1998). Interfacial heat transfer rate, on the other hand, is

$\dagger$ Email address for correspondence: lianshen@jhu.edu 
mainly controlled by air-side processes, and water-side fluid motion plays an essential role in the variation of temperature at the water surface associated with the cool skin.

In the past century, considerable research has been conducted to model the hydrodynamic process of surface renewal in the transport of passive scalars. Many conceptual models have been proposed (e.g. Higbie 1935; Danckwerts 1951; Harriott 1962). Effects of macro-scale eddies (Fortescue \& Pearson 1967) and Kolmogorovscale eddies (Banerjee, Scott \& Rhodes 1968; Lamont \& Scott 1970) on surface renewal have been modelled. To describe the dominant role played by upwelling (the strength of which is quantified by surface divergence) on surface renewal, Chan \& Scriven (1970) used irrotational stagnation flow as the principle element of advection action to obtain an analytical solution of the gas concentration. Using simulation for the advection-diffusion of scalars in the surface-normal direction with randomly varying advection velocity, Hanratty and co-workers (McCready \& Hanratty 1984; McCready, Vassiliadou \& Hanratty 1986) showed that, unlike the solid wall case where only low-frequency velocity fluctuations contribute to the scalar transfer (Campbell \& Hanratty 1982, 1983), in the free surface case velocity fluctuations of all frequencies are important and the gas transfer rate is related to the root-mean-square (r.m.s.) of surface divergence. Brumley \& Jirka (1988) obtained the Lagrangian time spectrum of surface divergence on the basis of the rapid distortion theory analysis of Hunt \& Graham (1978). The integration of the surface divergence spectrum was simplified by Banerjee (1990) to yield a surface divergence model for interfacial gas transfer, which was shown by Banerjee, Lakehal \& Fulgosi (2004) to agree well with experimental and direct numerical simulation (DNS) data.

Further improvement of the prediction capability for the interfacial scalar transfer requires a better understanding of the turbulent mixing and molecular diffusion processes. Recent developments in numerical simulation and experimental measurement have made it possible to obtain high-resolution data of flow and scalar fields. For example, DNS and large-eddy simulation (LES) of scalar transport in turbulent free-surface flows have been performed by e.g. Komori et al. (1993), Handler et al. (1999), Shen, Triantafyllou \& Yue (2001), Lakehal et al. (2003), Nagaosa \& Handler (2003), Magnaudet \& Calmet (2006), Hasegawa \& Kasagi (2008, 2009) and Khakpour, Shen \& Yue (2011). From such numerical simulation techniques, a detailed three-dimensional description of flow and scalar fields evolving in time can be generated. Similarly, high-resolution measurements have been obtained by Rashidi, Hetsroni \& Banerjee (1991), Chu \& Jirka (1992), Münsterer \& Jähne (1998), Tamburrino \& Gulliver (2002), Atmane \& George (2002), Garbe, Schimpf \& Jähne (2004), McKenna \& McGillis (2004), Zappa et al. (2004), Tamburrino, Aravena \& Gulliver (2007) and Herlina \& Jirka (2008), among many others. These studies have provided rich information on the flow and scalar fields and new insights into the process of scalar transport.

In this paper, we present a simulation-based study on the statistics of passive scalars in surface renewal. We choose turbulent open channel flow as the canonical problem, which has been widely used in DNS/LES and laboratory studies. We use DNS as a research tool (Moin \& Mahesh 1998) to focus on the fundamental characteristics of the scalar and flow fields related to the surface renewal. The wave effect is not a subject of the present study, and we intentionally keep the surface flat. Restricted free-surface vertical motion is a reasonable approximation for typical open channel flows with small Froude numbers in laboratory (see e.g. the analysis in Borue, Orszag $\&$ Staroselsky 1995). The present paper focuses on the use of Lagrangian tracing of surface fluid particles (Kermani \& Shen 2009) to directly quantify surface age 


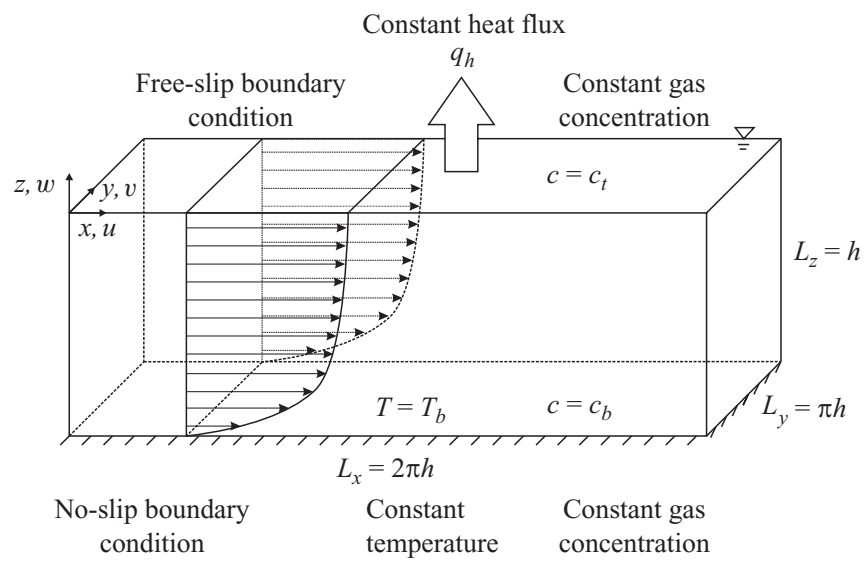

Figure 1. Computational geometry and definition sketch for transport of passive scalars (heat and gas) in open channel turbulent flow. Boundary conditions for flow, heat and gas are shown.

associated with the surface renewal process for a comprehensive study of scalar statistical properties. Different roles of turbulent advection and molecular diffusion for heat and gas at different stages during and after surface renewal are elucidated, based on which various approaches for surface age quantification are analysed. Using tools of probability density (p.d.f.) analysis, a clustering procedure based on the expectation-maximization algorithm, and nonlinear regression, we are able to gain further insight into the characteristics of heat and gas transfer at the free surface.

This paper is organized as follows. In $\S 2$, we introduce the problem definition and the numerical method, and provide an overview of the flow and scalar fields. In $\S 3$, we investigate surface renewal and quantification of surface age. In $\S 4$, we discuss the statistics of scalar features at the surface. Finally, $\S 5$ presents conclusions.

\section{Problem definition and overview of flow and scalar fields}

\subsection{Problem introduction and mathematical formulations}

We consider as a canonical problem a turbulent open channel flow shown in figure 1 . A body force in the streamwise direction drives the flow. The problem is periodic horizontally. In the vertical direction, the two flow boundary conditions are, namely no $\operatorname{slip}(u=v=w=0)$ at the channel bottom and free $\operatorname{slip}(\partial u / \partial z=\partial v / \partial z=w=0)$ at the channel top resembling a flat unsheared free surface. In this study, DNS is performed to simulate fluid motion. Unless indicated otherwise, the mean free-surface velocity $u_{f}$ is used to normalize velocities, and the depth of the channel $h$ is used to normalize lengths. The normalized Navier-Stokes equations and continuity equation are written as

$$
\begin{gathered}
\frac{\partial \boldsymbol{u}}{\partial t}+\boldsymbol{u} \cdot \nabla \boldsymbol{u}=\frac{1}{R e} \nabla^{2} \boldsymbol{u}-\nabla p+f_{\text {body }} \boldsymbol{i}, \\
\nabla \cdot \boldsymbol{u}=0,
\end{gathered}
$$

where $R e=u_{f} h / v$, with $v$ being the kinematic viscosity; $p$ is the dynamic pressure normalized by $\rho u_{f}^{2}$, with $\rho$ being the fluid density; $f_{b o d y}$ is the body force in the streamwise direction.

The heat and gas are modelled as passive scalars in the present study, i.e. convection due to the buoyancy effect is considered to be small compared with shear turbulence, which is a legitimate assumption for many open channel flows (see e.g. Handler et al. 
1999). There is no coupling between heat and gas transfer. The advection-diffusion equation for scalars is

$$
\frac{\partial f}{\partial t}+\boldsymbol{u} \cdot \nabla f=D \nabla^{2} f .
$$

In the above equation, $f$ can be the non-dimensional gas concentration $c$ or temperature $T$. In the case of gas, $D$ is $1 /(\operatorname{ReS} C)$, where $S c$ stands for the Schmidt number, defined as the ratio of kinematic viscosity to molecular diffusivity. In the case of temperature, $D$ is $1 /(\operatorname{Re} P r)$, where $P r$ stands for the Prandtl number, defined as the ratio of kinematic viscosity to thermal diffusivity. The normalized forms of gas concentration and temperature and their boundary conditions are discussed below.

For the transfer of gas and heat across the air-water interface, there exists resistance on both the air-side and the water-side. A study on the partition of the transfer (Liss \& Slater 1974) shows that their relative importance is determined by the molecular diffusivity of the scalar in air and water and the solubility of the scalar in water. As shown in Jähne \& Haußecker (1998), the flux rate of heat is controlled by air-side processes, while for most gases the flux is water-side controlled (results summarized in their figure 2). In numerical studies that simulate the water-side only, an approximation of the free-surface boundary conditions for the scalars is necessary. Handler et al. (1999) considered two cases, one with a Dirichlet condition for the scalar at the surface, and the other with a Neumann condition. Although in both cases the scalars were called 'temperature' for simplicity in their paper, they also pointed out that the first case actually applies to gas transfer and they stated that 'an excellent approximation is that the gas concentration in the air and at the free surface remains constant'. They also stated that the second case applies to heat transfer because 'the heat flux, which is controlled by the humidity and the wind, can be assumed to be held fixed to a close approximation in most situations'. Furthermore, they showed that the surface flux field of their first case is much more intermittent than the surface concentration field of their second case, the effect of microscale is more significant in the first case (which has been confirmed in our study; since our result is very similar to theirs, it is not shown here), and they validated their results by comparison with observations from measurement. Recently, Liu et al. (2009) simulated scalar transport in coupled air-water boundary layers and confirmed the conclusions of above studies regarding the applicability of the boundary conditions for the two types of scalars.

Therefore, for the simulation of gas transport in the present study, Dirichlet boundary conditions are used at the free surface and at the bottom. (We note that in some laboratory experiments using tanks, there is no gas flux at the tank bottom; the case of zero flux at the bottom, i.e. the Neumann bottom condition, is discussed in Appendix A.) Gas concentration is normalized as $c=\left(c^{*}-c_{b}^{*}\right) /\left(c_{t}^{*}-c_{b}^{*}\right)$, where the superscript ' $*$ ' stands for the dimensional value, and the subscripts ' $t$ ' and ' $b$ ' stand for values at the top free surface and the bottom wall, respectively. After this normalization, the boundary conditions for gas are $c_{t}=1$ at the free surface and $c_{b}=0$ at the bottom; the gas flux at the free surface is $q_{g}=\left.D(\partial c / \partial z)\right|_{z=0}$. For temperature, a Neumann boundary condition at the free surface and a Dirichlet boundary condition at the bottom are used in our simulation. The normalized temperature is $T=-\left(T^{*}-T_{b}^{*}\right) k /\left(q_{h}^{*} h\right)$, where $T_{b}^{*}$ is the temperature at the bottom of the channel, $k$ is the thermal conductivity, and $q_{h}^{*}$ is the heat flux at the free surface that is set to be a constant in this study. This normalization simplifies the boundary conditions as $\partial T / \partial z=-1$ at the free surface and $T=0$ at the bottom of the channel. For simplicity, we set the non-dimensional surface heat flux to be $q_{h}=\left.D(\partial T / \partial z)\right|_{z=0}$. 
To summarize, the boundary conditions for gas concentration and temperature are

$$
\left.\begin{array}{l}
c=0, \quad T=0, \quad \text { at } \quad z=-1, \\
c=1, \quad \frac{\partial T}{\partial z}=-1, \quad \text { at } \quad z=0,
\end{array}\right\}
$$

which are based on the different air-side and water-side transport processes for gas and heat. In previous numerical studies (e.g. Komori et al. 1993; Handler et al. 1999; Liu et al. 2009), these boundary conditions have been shown to capture the salient features of gas and temperature at the water surface.

\subsection{Numerical method and computational parameters}

To simulate (2.1)-(2.3), we use the fractional step method introduced by Kim \& Moin (1985). For time integration, we use a second-order explicit Adams-Bashforth scheme for the convective terms and a second-order implicit Crank-Nicholson scheme for the viscous terms. For spatial integration, we implement a sixth-order finite-difference scheme in the horizontal directions and a second-order one in the vertical direction with a vertically staggered grid. The details are provided in Kermani (2010).

In this study, the friction Reynolds number of the open channel turbulent flow, $R e_{*}=u_{*} h / v$, is defined based on the wall friction velocity at the bottom $u_{*}$, channel depth $h$ and kinematic viscosity $v$. We use the value $R e_{*}=300$ in our DNS, which leads to the value of the Reynolds number based on the free-surface velocity $R e=u_{f} h / \nu=5778$. Prandtl and Schmidt numbers with the values of 0.71, 1, 2, 4 and 8 are used.

The computational domain size is $L_{x}=2 \pi$ in the streamwise direction, $L_{y}=\pi$ in the spanwise direction, and $L_{z}=1$ in the vertical direction, all scaled by the channel depth $h$. To ensure that the domain used in the simulation is sufficiently large, we analysed the two-point correlation in the streamwise and spanwise directions for flow and scalar properties, and confirmed that the correlation coefficients at the half domain distance are sufficiently small. A uniform grid size is used in the horizontal directions. An uneven grid is used in the vertical direction and is clustered close to the boundaries. The grid number is $128^{3}$ or $256^{3}$, depending on the values of $\mathrm{Pr}$ and Sc. Validation of our simulation result and comparison with experimental and other numerical results are shown in $\S 2.3$.

The simulation starts with prescribed mean velocity and scalar concentration profiles seeded by noise. Turbulence is then developed dynamically in the simulation. To obtain statistical steady turbulence, we apply a constant body force in the flow to counter the friction at the channel bottom. This is equivalent to having a mean pressure gradient in the streamwise direction. After the flow and scalar fields have fully developed, as indicated by quasi-stationarity in the turbulence statistics, we continue the simulation for $1000 u_{f} / h$ (about 280 large-eddy turnover time) to obtain data for time averaging.

We remark that the values of $R e, S c$ and $P r$ are limited in DNS by the requirement of resolving all the turbulence scales without modelling. Like other DNS of freesurface scalar transport in the literature as reviewed in the Introduction, our DNS does not seek a direct solution of the problems in real-world applications. Instead, the DNS is used as a research tool (Moin \& Mahesh 1998) to reveal the fundamental characteristics of gas and heat transport at a free surface, which can then be used as a physical basis for the development of improved models and parameterizations in future studies. The value of $R e$ is consistent with the DNS studies in the literature and is comparable to typical small-scale laboratory measurements of open channel 
(a)

$\langle T\rangle$

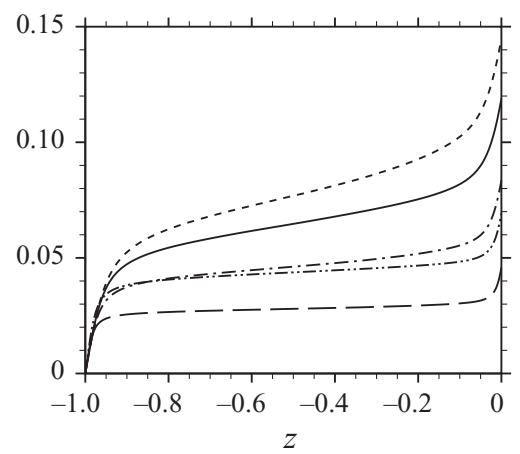

(c)

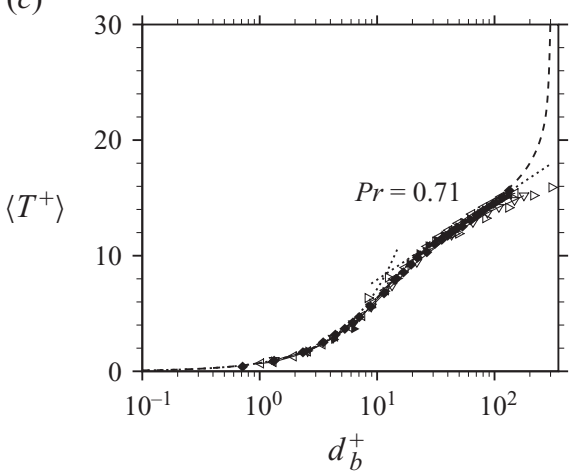

(b)

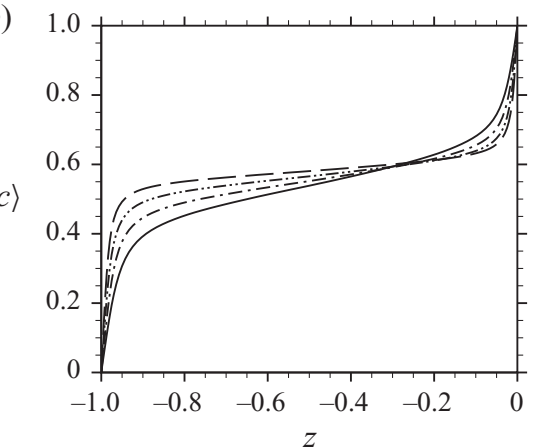

$(d)$

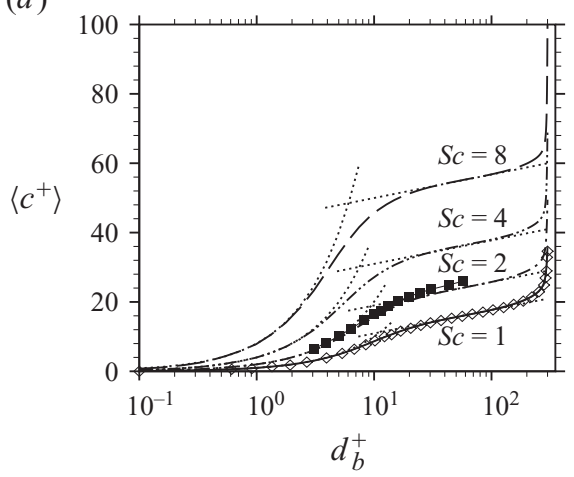

FIGURE 2. Mean temperature and gas concentration profiles in $(a, b)$ the global coordinate and $(c, d)$ the local coordinate near the bottom wall. The present results are denoted by --- , $P r=0.71 ;-, P r=S c=1 ;-\cdot-, P r=S c=2 ;-\cdots-, P r=S c=4 ;--, P r=S c=8$. Plots $(c)$ and $(d)$ show the comparison with the experimental results of Johnk \& Hanratty $(1962)(\triangleright)$, Kader (1981) ( $\$$ ) and Zhu \& Antonia (1993) ( $\diamond)$ with $\operatorname{Pr}=0.71$; the numerical

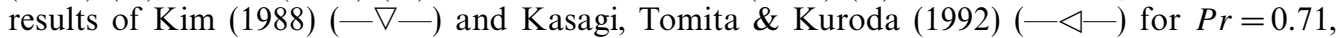
Nagaosa \& Handler (2003) $(-\diamond-)$ for $S c=1$, and Handler et al. (1999) (-口-) for $S c=2$; and the law of the wall $(\cdots \cdots)$.

flows (Kermani 2010). The value of $\operatorname{Pr}$ for heat in nature can be accounted for by DNS. For most gases in nature, on the other hand, the value of $S c$ is much larger than what is permitted by DNS, and proper scaling is needed to estimate the gas transfer rate (as explained in more detail in $\$ 2.3$ ). Nevertheless, the limitation in $S c$ does not cause a concern for the main topic of this paper, surface renewal, which is a hydrodynamic process. The scalars are assumed to be passive, and thus the flow patterns are not affected by scalar concentration.

\subsection{Results overview and validation}

In this section, we present an overview of the scalar results and their validation. Extensive comparison of our simulation results with those in the literature has been performed in Kermani (2010) to validate our simulation. Because this type of open channel flow has been successfully modelled in numerous other DNS studies and our simulation approach is similar, the agreement in the flow field results is as expected and we omit the comparison results of the velocity field for space consideration. The comparison of the scalar field, on the other hand, is less reported in the literature and we present it below.

Figure 2 shows the mean temperature and gas concentration profiles. Here and hereafter, the mean value of a quantity $f(x, y, z)$ is expressed as $\langle f\rangle(z)$, where $\langle\cdot\rangle$ 
denotes averaging over the $(x, y)$-plane because the present problem is statistically homogeneous in the horizontal directions. Fluctuation of the quantity is expressed as $f^{\prime}(x, y, z, t)=f-\langle f\rangle$, and its r.m.s. value is denoted as $f_{r m s}(z)=\left\langle f^{\prime 2}\right\rangle^{1 / 2}$. The results are further averaged over 280 large-eddy turnover times to improve the accuracy of the statistics. When a global view is needed, the results are presented in the global coordinate $z \in[-1,0]$. When detailed structures near the boundaries are examined, we use the local coordinate in terms of the distances from the bottom wall $d_{b}=(z+1)$ and from the top free surface $d_{t}=-z$.

Figure 2(a,b) shows the global view for $\operatorname{Pr}=0.71$ and $\operatorname{Pr}=S c=1,2,4$ and 8 . It is noted that at the free surface, the gas concentration is fixed, while for the temperature it is the gradient being specified (see (2.4)). As $P r$ or $S c$ increases, the scalar diffusive sublayer becomes thinner. Between the top free surface and the bottom solid wall, the diffusive sublayer is thinner at the former. This is due to the fact that according to the Taylor series expansion, $w \propto d_{t}$ near the free surface and $w \propto d_{b}^{2}$ near the solid wall. As a result, as the distance from the boundary increases, turbulent transport increases more rapidly near the free surface and thus its diffusive sublayer is thinner.

Comparison of the present result with previous studies is shown in figure $2(c, d)$ for the near-bottom region in the local coordinate $d_{b}^{+}$(the near-surface region is compared in figure 4). Here, the superscript ' + ' denotes normalization by the friction velocity $u_{*}$ and the viscous length scale $v / u_{*}$. For scalars, the superscript ' + ' indicates that the temperature and gas concentration are normalized by the friction temperature $T_{*}=\left|\left\langle q_{h}\right\rangle\right| / u_{*}$ and the friction gas concentration $c_{*}=\left\langle q_{g}\right\rangle / u_{*}$, respectively. For the heat transfer problem with $\operatorname{Pr}=0.71$, extensive measurement (using air) and simulation data exist in the literature. A few representative data results are included in figure 2(c) to show that the present result agrees with the results obtained by other researchers. For $S c=1$ and 2, only simulation results are available in the literature, which again are closely matched by the present data (figure $2 d$ ).

The results in figure $2(c, d)$ show that at the channel bottom, the law of the wall is satisfied with $\left\langle c^{+}\right\rangle=S c d_{b}^{+}$and $\left\langle c^{+}\right\rangle=\left(1 / k_{\theta}\right) \ln d_{b}^{+}+B_{\theta}$. (Note that at the bottom that is away from the surface, the behaviour of gas and temperature is indiscernible if $\operatorname{Pr}=S c$, and we discuss only one of them.) The slope $1 / k_{\theta}$ is $1 / 0.34$, which is determined by $R e$ and is independent of $S c ; B_{\theta}$ varies from 1.15 to $4.15,12.2,24.2$ and 43.2, for $P r=0.71$ and $S c=1,2,4$ and 8 , respectively. The independence of $1 / k_{\theta}$ with respect to $S c$ and the variation of $B_{\theta}$ with $S c$ were observed by $\mathrm{Na}$, Papavassiliou \& Hanratty (1999) and Schwertfirm \& Manhart (2007). An estimate for the thickness of the diffusive sublayer can be obtained using the intersection point of the linear and the logarithmic curves (see e.g. Schwertfirm \& Manhart 2007). Using the present result, the thickness of the diffusive sublayer is fitted to $11.1 S c^{-0.28} v / u_{*}$ for the range of $S c=0.71-8$, which is in agreement with the empirical equation of Schwertfirm \& Manhart (2007) of $11.5 S c^{-0.29} v / u_{*}$ that was obtained for the range of $S c=$ 1-49.

Figure 3 shows the r.m.s. profile of scalar fluctuations. Figure $3(a, b)$ shows that, as seen in the velocity fluctuations, $T_{r m s}^{+}$and $c_{r m s}^{+}$have local maxima near the bottom. At the free surface, the fixed flux boundary condition makes the peak of $T_{r m s}^{+}$located at the surface, while the Dirichlet boundary condition makes the peak of $c_{r m s}^{+}$located at a short distance below the surface. As $S c$ increases, the near-surface peak of $c_{r m s}^{+}$ increases and moves towards the surface. Magnaudet \& Calmet (2006) found that the location of the near-surface maximum of $c_{r m s}^{+}$satisfies $S c^{0.5} d_{t}^{+}=15$, which is in agreement with the present result $S c^{0.5} d_{t}^{+}=15.5$. Near the bottom, we compare the scalar fluctuation intensity with the previous measurements and simulations in a way 

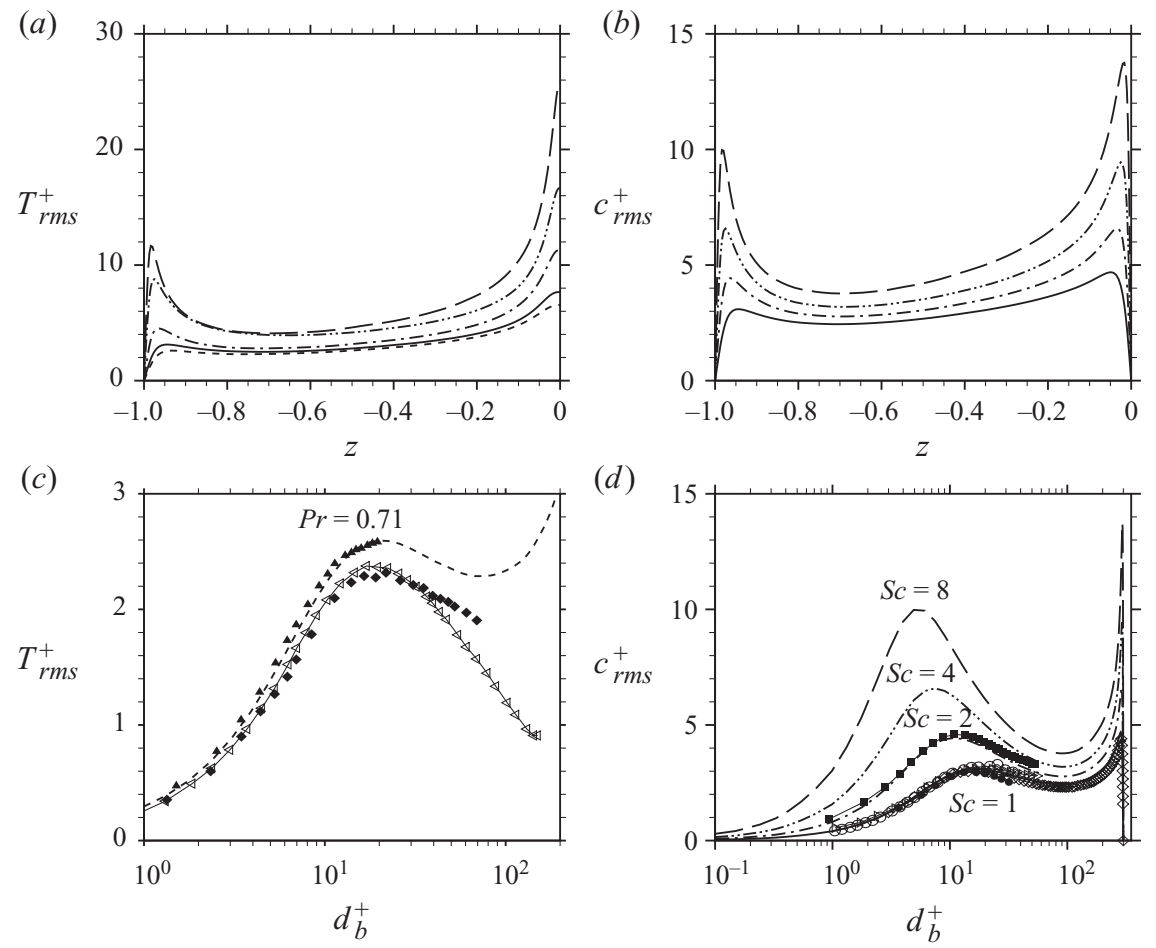

FIGURE 3. Profiles of r.m.s. of temperature and gas concentration fluctuations in $(a, b)$ the global coordinate and $(c, d)$ the local coordinate near the bottom wall. The present results are denoted by,$--- \operatorname{Pr}=0.71 ;-, P r=S c=1 ;-\cdot-, \operatorname{Pr}=S c=2 ;-\cdots-, \operatorname{Pr}=S c=4 ;--$, $\operatorname{Pr}=S c=8$. Plots $(c)$ and $(d)$ show the comparison with the experimental results $(P r=0.71)$ of Nakajima et al. (1980) (A) and Zhu \& Antonia (1993) (\$); and the numerical results of Kasagi et al. (1992) (- - -) for $P r=0.71$, Lyons, Hanratty \& Mclaughlin (1991) (- $\square-)$, Na \& Hanratty (2000) (—o-), Lakehal et al. (2003) (-— - ), and Nagaosa \& Handler (2003) $(-\diamond-)$ for $S c=1$, and Handler et al. (1999) (-口-) for $S c=2$.

similar to the preceding discussion of the mean profiles. Figure $3(c, d)$ shows the agreement.

We next examine the near-surface region in more detail. It should be noted that the measurement of scalars is more challenging near a free surface than near a solid wall. As a result, it is difficult to find suitable experimental data in the vicinity of the free surface to compare with the present simulation. Fortunately, there are new experimental results that have been obtained using oscillating grid-stirred tanks. While the data appear scattered due to the complexity of the problem, the results provide important information, as explained in more detail below. Figure 4 shows the comparison between these data and our DNS results.

We first discuss the mean gas concentration near the free surface shown in figure 4(a). The comparison of our open channel flow simulation with the oscillating grid-stirred tank measurements requires proper normalization. Following Magnaudet \& Calmet (2006), we normalize the gas concentration by the difference between the concentration at the surface $c_{t}$ and that in the bulk flow $c_{b u l k}$ (for which we choose the channel centre), $\Delta c=c_{t}-c_{\text {bulk }}$. The depth is normalized by the thickness of the diffusive surface layer, $\delta_{c, f s}$, which is quantified as $\Delta c /\left.(\partial\langle c\rangle / \partial z)\right|_{z=0}$ (Jähne \& Haußecker 1998) in the present study. The surface renewal model predicts $\left\langle c-c_{b u l k}\right\rangle / \Delta c=\exp \left(-d_{t} / \delta_{c, f s}\right)$ 

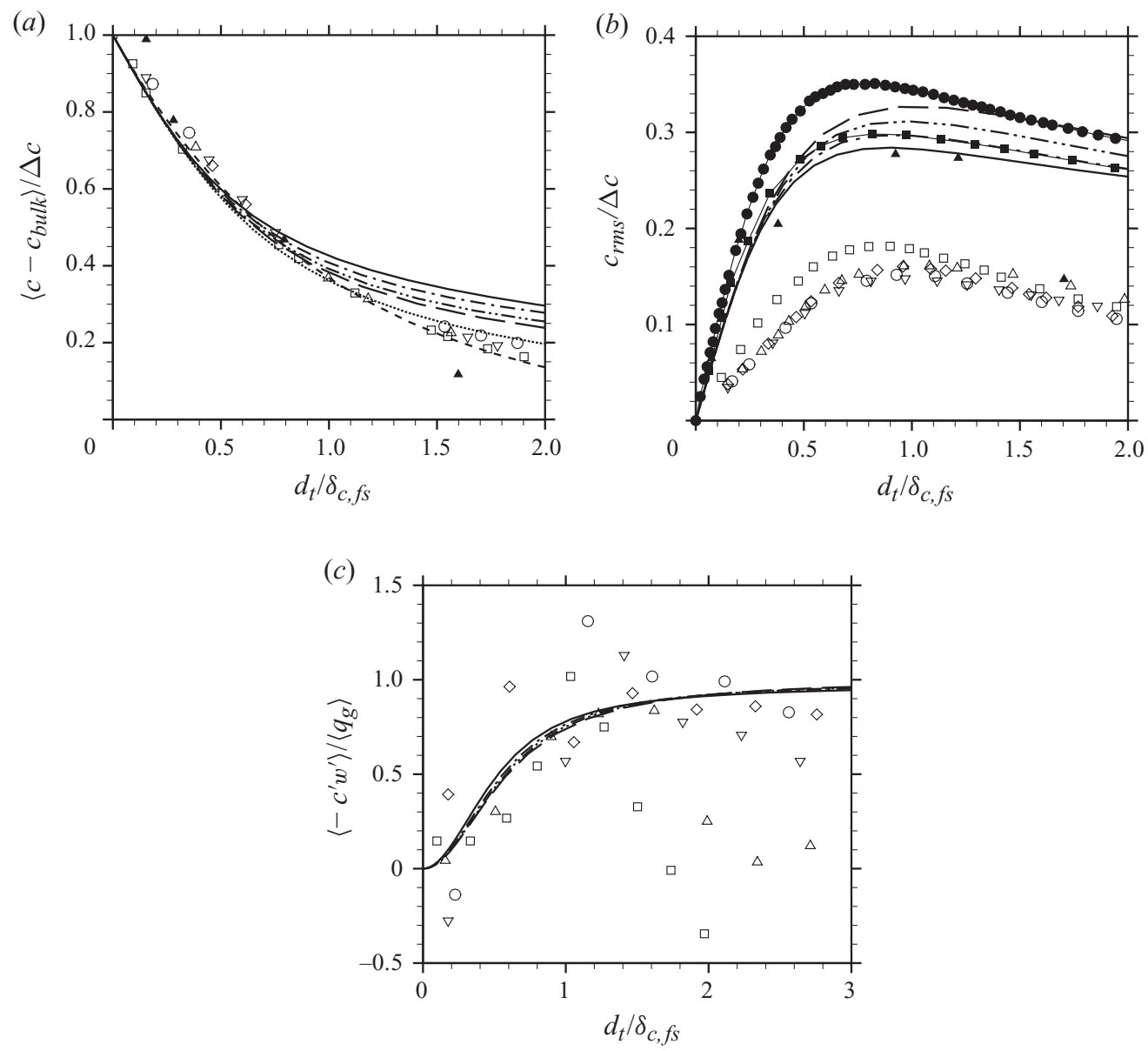

FIGURE 4. Near-surface profiles of $(a)$ mean gas concentration, $(b)$ r.m.s. of gas concentration fluctuations, and $(c)$ turbulence gas flux. The present results are denoted by,$- S c=1$; $-\cdot-, S c=2 ;-\cdots-, S c=4 ;--, S c=8$. Shown are the comparison with the experimental results of Herlina \& Jirka (2008) with their cases of $R e=260(\square), R e=390(\triangle), R e=520(\nabla)$, $R e=650(\diamond)$ and $R e=780(\bigcirc)$; the experimental result of Atmane \& George (2002) $(\Delta)$ with $R e=230$; the numerical results of Handler et al. (1999) (- - ) and Magnaudet \& Calmet (2006) (- - ) ; and the predictions of the surface renewal $(----)$ and small eddy $(\cdots \cdots \cdots)$ models.

and the small-eddy model predicts $\left\langle c-c_{b u l k}\right\rangle / \Delta c=(2 / \pi) \operatorname{arccotg}\left((\pi / 2) d_{t} / \delta_{c, f s}\right)$ (Jähne \& Haußecker 1998; Herlina \& Jirka 2008). Therefore, in the results reported by Herlina \& Jirka (2008), $\delta_{c, f_{s}}$ is defined as the location where the mean gas concentration is reduced to $1 / e$ of the surface value. Figure $4(a)$ shows that our simulation agrees with the measurement of Herlina \& Jirka (2008) for $d_{t} / \delta_{c, f s}<0.8$. Also plotted in the figure are the formulations based on the surface renewal model and the small-eddy model. It should be noted that as the depth increases, the deviation of our result from the results obtained by other researchers is not surprising because the open channel flow in the present DNS introduces different features in the region below.

Figure 4(b,c) shows the comparison of gas concentration fluctuation intensity and turbulence flux of gas. It is unclear to us why in figure $4(b)$ the normalized gas 
concentration fluctuation from Herlina \& Jirka (2008) is lower than the results of other studies and the present one, although it is noted that the trends of variation are consistent. The differences in the flow set-up may play a role. To have a clearer comparison, it would be desirable to simulate a forced homogeneous turbulence flow (instead of the present open channel flow) to match the flow condition of the stirredgrid tank in Herlina \& Jirka (2008), probably under various surfactant conditions (Shen, Yue \& Triantafyllou 2004), but this is beyond the scope of the present paper. Figure 4(c) shows that the measured turbulence flux has large fluctuations. (As Herlina \& Jirka 2008 pointed out, the turbulence flux normalized by the total flux should be between zero and one everywhere and should approach one as the depth increases.) There is closer agreement of our results with other numerical and theoretical work.

Finally, we discuss the mean gas transfer coefficient $K=(D / \Delta c) \partial\langle c\rangle / \partial z$ at the free surface. For $S c=1-8$ in the present study, $K / u_{*}$ is fitted to $0.0611 S c^{-0.451}$. (For heat with $\mathrm{Pr}=0.71-8$, it is found to be $0.0604 \mathrm{Pr}^{-0.450}$.) This result is approximately consistent with the $S c^{-1 / 2}$-scaling for a clean free surface (Jähne \& Haußecker 1998) (the deviation is probably caused by the low $S c$ that is used due to the DNS limitations). In reality, if the surface is contaminated by surfactants, the scaling would be changed to be close to $S c^{-2 / 3}$.

The mean gas transfer coefficient is often related to surface divergence $\beta=\partial u / \partial x+$ $\partial v / \partial y$ by $K S c^{1 / 2}=A \beta_{r m s}^{1 / 2}$. The coefficient $A$ is found to be in the range of $(0.41,0.45)$ for $S c=1-8$ in the present study. For shear-free free surfaces, the LES of Magnaudet \& Calmet (2006) and the hybrid DNS/LES of Hasegawa \& Kasagi (2009) suggested values of 0.6 and 0.44 , respectively. We evaluate $A$ in the laboratory measurements of McKenna \& McGillis (2004) (based on figure 6 in their paper) and Herlina \& Jirka (2008) (based on figure 9 in their paper) to be in the ranges of $(0.37,0.64)$ and $(0.26,0.33)$, respectively. The $A$ value is also estimated to be in the range of $(0.22,0.42)$ for the experimental study of Tamburrino et al. (2007) (based on their figure 5). Sugihara \& Tsumori (2005) reported a value of 0.3 in their measurements. Moreover, the study of Banerjee et al. (2004) found that the surface divergence model can be applied to sheared free surfaces, which is supported by the simulations of Lakehal et al. (2008a) and Lakehal, Fulgosi \& Yadigaroglu (2008b). This observation is also verified in the experimental work of Turney, Smith \& Banerjee (2005) with $A$ being 0.45 and in the recent numerical simulation of Hasegawa \& Kasagi (2009) with $A$ being 0.40 for $S c$ of 1 and 100 .

\section{Surface renewal and statistics of surface age}

\subsection{Structure of surface renewal}

The hydrodynamic processes of upwelling and downwelling play an essential role in scalar transport near the free surface. In open channel flows, upwelling events at the free surface are associated with coherent vortical structures originating from the near bottom region (see e.g. Komori, Murakami \& Ueda 1989; Rashidi et al. 1991; Nagaosa \& Handler 2003). The upwelling increases interfacial transfer by replacing fluid close to the surface with fresh fluid from the bulk flow (surface renewal).

Figure 5(a) shows three upwellings near the figure centre, which are characterized by diverging flow with stretched surface. In the case of downwelling shown in the upper right corner of figure 5(a), the surface flow converges. Therefore, positive and negative values of surface divergence $\beta$ can be used to detect upwellings and downwellings, respectively. In practice, a threshold of $\beta$ is used to identify large upwelling and downwelling events (more discussion is provided in $\S 3.2 .1$ ). 

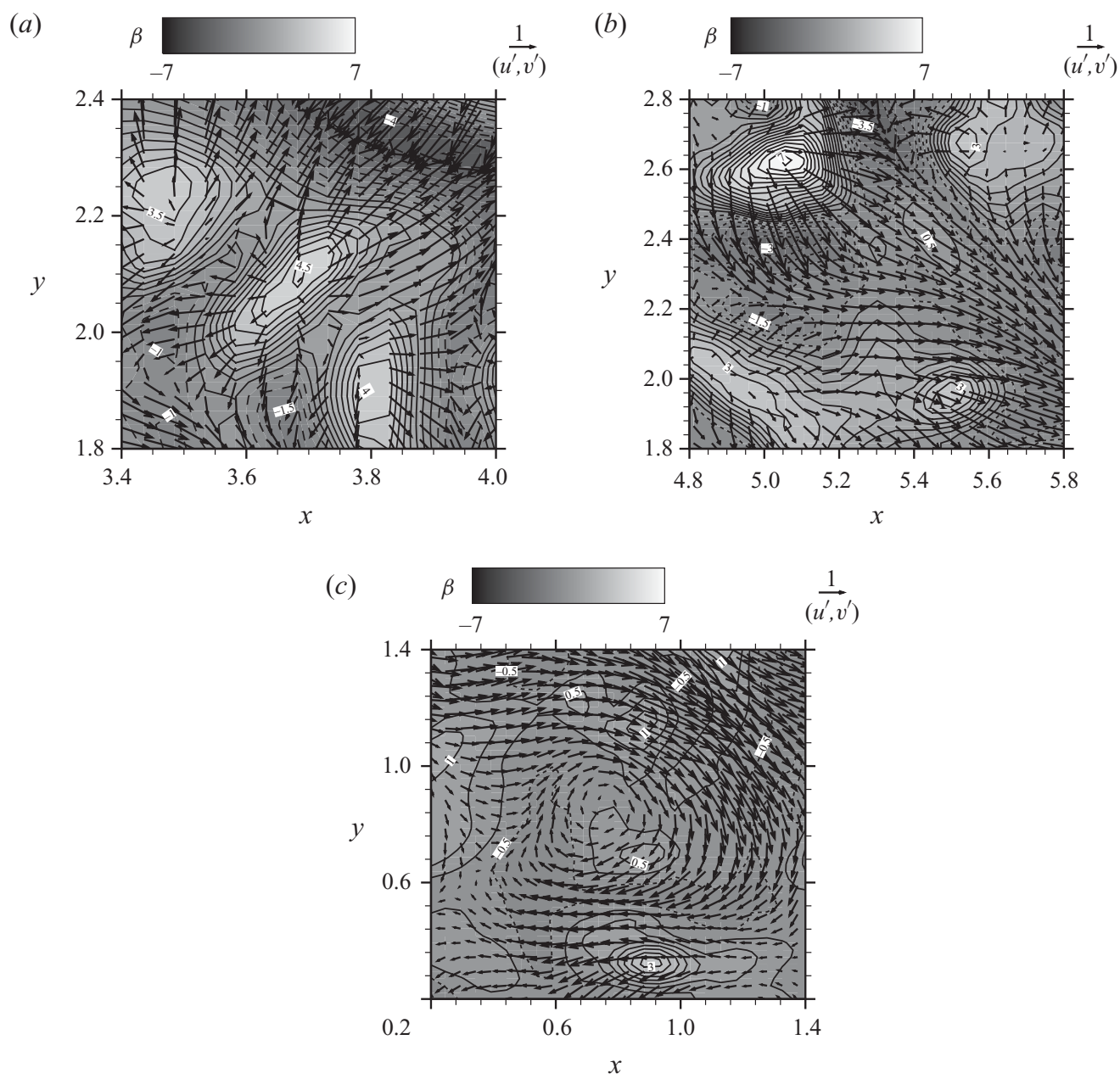

FiguRE 5. Free surface features of ( $a$ ) strong upwellings; $(b)$ a weak upwelling, marked by 'A' in the figure, near strong upwellings; and $(c)$ an upwelling near a surface-connected vortex. Vectors represent fluctuations of velocity $\left(u^{\prime}, v^{\prime}\right)$ and contours represent surface divergence $\beta$. All variables are normalized by their r.m.s. values. In the contour plots, the contour increment is 0.5 and dashed contour lines represent negative values.

While figure 5(a) shows a clear picture of several strong upwellings with flow radiating from the upwelling centres, there are other forms of upwelling that are more complex. Figure $5(b)$ shows a weak upwelling near a strong upwelling. In this case, surface flow is mainly induced by the strong upwelling. Some fluid particles are pushed over the weak upwelling. As they pass the weak upwelling, a surface renewal occurs. Figure 5(c) shows an upwelling near a strong surface-connected vortex (for the dynamics of the vortex, see e.g. Handler et al. 1993; Pan \& Banerjee 1995; Zhang, Shen \& Yue 1999). In this case, the advection induced by the surface-connected vortex pushes some fluid particles over the upwelling as well. Therefore, the complex nature of free-surface turbulence complicates the upwelling process and its quantification. In $\S 3.2$, we use Lagrangian tracing of surface fluid particles to quantify the surface renewal, and the subsequent interfacial scalar transfer processes the fluid particles experience. 

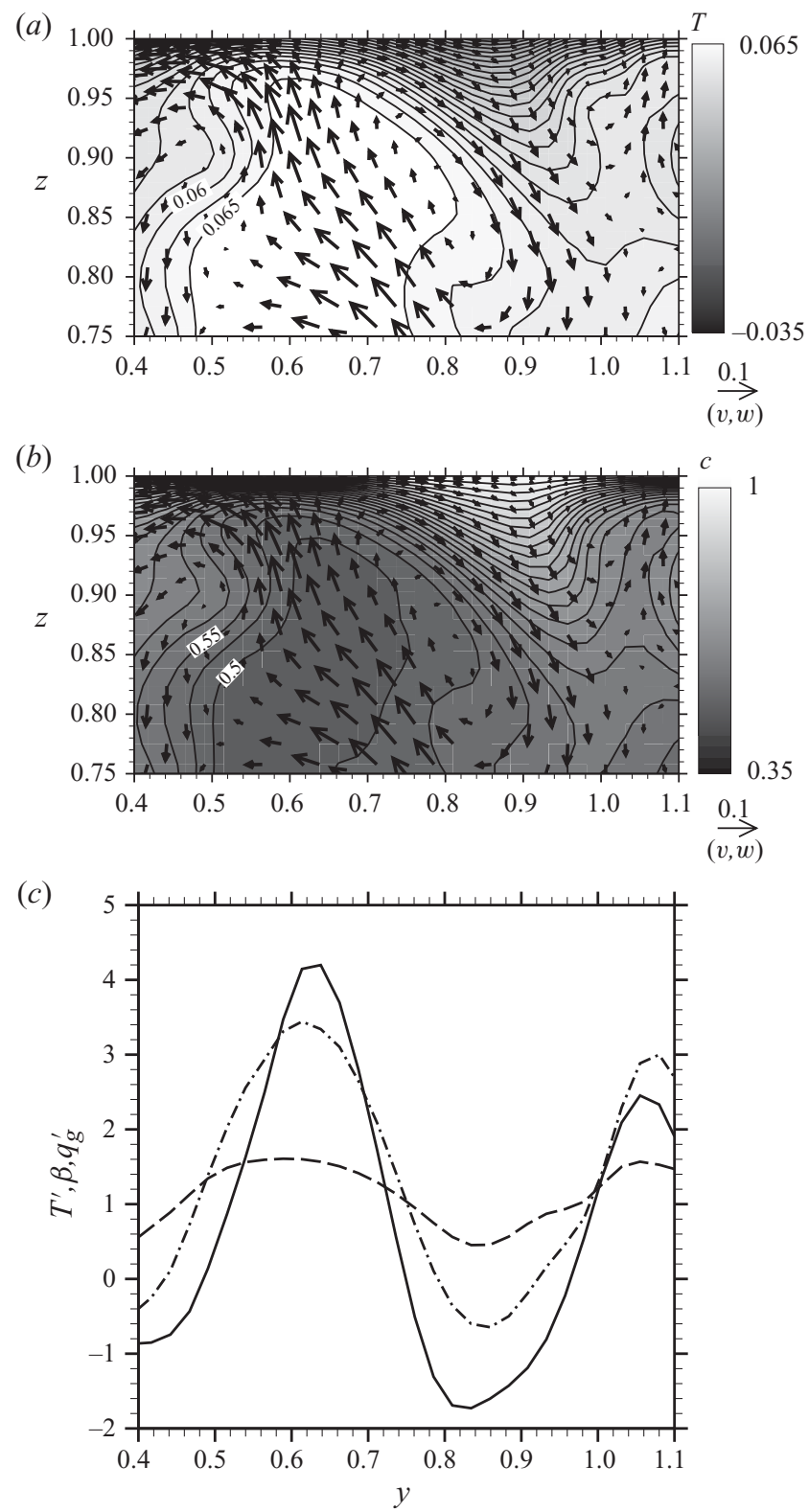

FIGURE 6. Vertical section of flow with contours of $(a)$ temperature $T$ and $(b)$ gas concentration $c$. Vectors represent velocity $(v, w)$. (c) Surface profiles of: - , surface divergence $\beta$; -- , temperature fluctuation $T^{\prime} ;--$, gas flux fluctuation $q_{g}^{\prime}$. In $(a), T$ is subtracted by mean surface temperature. In $(c)$, all quantities are normalized by their r.m.s. values.

Figure $6(a, b)$ shows an instantaneous vertical section of upwelling and downwelling with contours of temperature and gas concentration. The contours in the vertical section look similar between the two scalars, suggesting that gas and temperature respond to upwelling in a similar way. At the free surface, however, there exists significant difference between the gas flux and the temperature. Note that the freesurface boundary conditions for gas concentration and temperature (2.4) are Dirichlet and Neumann, respectively; as a result, it is the gas flux and the temperature that 
vary at the surface. Profiles of instantaneous fluctuations of gas flux, temperature and surface divergence along the free surface in the corresponding section are plotted in figure $6(c)$. It shows that over the upwelling and downwelling regions, the gas flux has large magnitude of variations together with the surface divergence. The variation in the surface temperature, in contrast, is relatively small. We emphasize that in figure 6(c), all fluctuation values have been normalized by their r.m.s. values at the free surface. Therefore, the difference between the surface temperature and the surface gas flux is not a scaling issue.

Upwelling increases interfacial transport by mixing the flow and pushing the mass and thermal boundary layers towards the surface to make them thinner. As the mass boundary layer becomes thinner, the gas flux $q_{g}=D \Delta c / \delta_{c, f s}$ increases immediately. We found that the correlation between $\beta$ and $q_{g}$ in upwelling areas is 0.70 . For the temperature, as the thermal surface layer thickness $\delta_{T, f s}$ becomes smaller, the time scale of heat diffusion $t=\delta_{T, f s}^{2} / D$ is reduced. Therefore, the temperature at the surface is also enhanced by upwelling. However, this process occurs over a molecular diffusion time scale, which is slower than the kinematic time scale of gas flux response (Handler et al. 1999).

\subsection{Quantification of surface age}

In the study of the effect of surface renewal on interfacial scalar transfer, it is essential to obtain information on surface age. In the literature, simple theories for surface age distribution have been proposed. They have been widely used in various applications, but have not been validated rigorously because of the difficulty in measuring surface age directly. The availability of extensive data set from DNS provides an opportunity for the investigation of surface age.

\subsubsection{Quantification of surface age using hybrid Lagrangian tracing and temperature method}

Because the surface age of a surface fluid particle is defined as the time elapsed since it last experienced an upwelling (surface renewal), Kermani \& Shen (2009) proposed to use Lagrangian tracing of fluid particles to obtain surface age directly. Note that only the fluid particles at the free surface need to be traced. Because of the kinematic free-surface boundary condition, all surface particles stay at the surface, which in the present case is flat. As a result, we can focus exclusively on the channel top, defined by $z=0$.

Let $\boldsymbol{u}_{s}(x, y ; t)$ represent the surface velocity at location $(x, y ; z=0)$ and time $t$; we have

$$
x_{1}=x_{0}+\int_{t_{0}}^{t_{1}} u_{s}(x, y ; t) \mathrm{d} t, \quad y_{1}=y_{0}+\int_{t_{0}}^{t_{1}} v_{s}(x, y ; t) \mathrm{d} t .
$$

Here, $\left(x_{0}, y_{0}\right)$ and $\left(x_{1}, y_{1}\right)$ are the locations of a surface particle at times $t_{0}$ and $t_{1}$, respectively. Equation (3.1) can be used for forward tracing $\left(t_{0}<t_{1}\right)$ and backward tracing $\left(t_{0}>t_{1}\right)$. In implementing (3.1) using DNS data, numerical accuracy should be ensured. As shown in figure 5, large gradients of $\boldsymbol{u}_{s}$ occur when strong upwellings, downwellings or vortical motions are present. As a result, small errors in the tracing may be significantly amplified later if the numerical scheme is not accurate. In this study, the surface data are stored frequently at a time interval of $0.35 \%$ of largeeddy turnover time. We use a fourth-order Runge-Kutta method to perform the time integration in (3.1). To find $\boldsymbol{u}_{s}$ at a location between the grid points, we use a sixth-order interpolation in space. Rigorous convergence tests have been performed (Kermani 2010). 


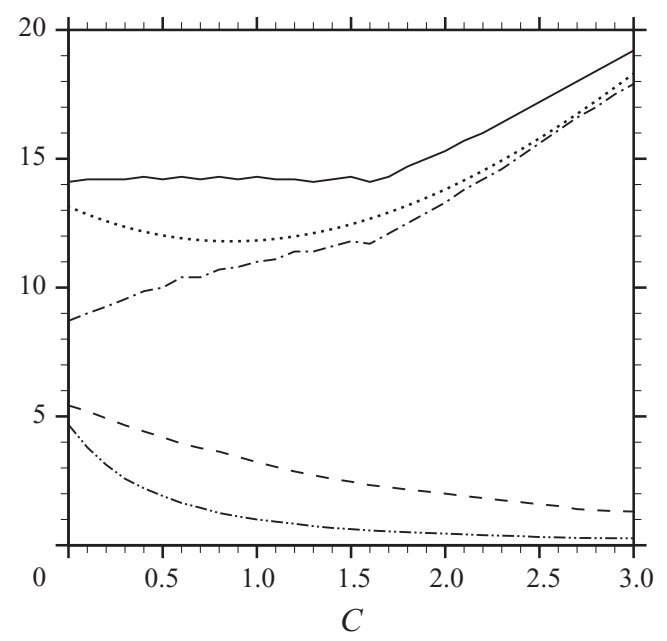

FIGURE 7. Variation of average surface age with the threshold value of $C$ used in the criterion for determining the time when surface parcels enter upwellings: $-\tau=\tau_{L}+\tau_{T}$; $-\cdot-, \tau_{L} ;--, \tau_{T} ; \cdots, \tau=\tau_{L}+\tau_{q_{g}} ;-\cdots-, \tau_{q_{g}}$.

Lagrangian tracing provides a means to directly measure the time a surface fluid particle takes to travel with respect to the surface renewal process. In particular, for a surface particle, the time elapsed since the last surface renewal it experienced is its surface age. In determining the surface age of a particle, one would like to trace it backwards in time until the 'birth time'. In practice, however, one has to stop tracing at some point within the originating upwelling region because the geometry of such regions is complex (figure 5) with a highly irregular evolution in time. To overcome this difficulty, Kermani \& Shen (2009) developed a hybrid Lagrangian tracing and temperature (HLTT) method in which the Lagrangian tracing is combined with a local temperature-based Eulerian approach: a surface particle is traced backwards in time for a duration of $\tau_{L}$ until it enters an upwelling region. Then the tracing stops and the temperature of the surface particle is used to obtain its Eulerian surface age $\tau_{T}$ at that location:

$$
\tau_{T}=\left(\frac{\Delta T}{2 q_{h}}\right)^{2} \pi D
$$

In the above equation, $\Delta T$ is the difference between the temperature of the surface particle and the bulk flow; heat diffusion is used to relate $\tau_{T}$ to $\Delta T$. The derivation of (3.2) is given in Appendix B. Finally, the surface age is obtained as $\tau=\tau_{L}+\tau_{T}$.

In the above hybrid method, upwelling areas are defined according to $\beta>C \beta_{r m s}$, with $C$ being a threshold and $\beta_{\text {rms }}$ the plane r.m.s. value of $\beta$. The choice of $C$ is not unique. Small $C$ may lead to the inclusion of surface diverging events too weak to be called upwellings (a special case is $C=0$ ), while large $C$ may select very strong upwellings only. We have tested various $C$ values and found that the result of $\tau$ is relatively insensitive to the choice of $C$. Figure 7 shows the variation of the average values of $\tau, \tau_{L}$ and $\tau_{T}$ with $C$. As $C$ increases, the switch from Lagrangian tracing to the use of (3.2) is delayed, and as a result $\tau_{L}$ increases while $\tau_{T}$ decreases. However, their summation changes little over the range of $C \in(0,1.6)$, indicating the robustness of this method. For large $C(>1.6), \tau$ increases to unrealistically large values because some upwellings are passed by the Lagrangian tracing and the particles keep travelling to earlier upwellings. In our study, we use $C=1$ as the criterion because for a wide 


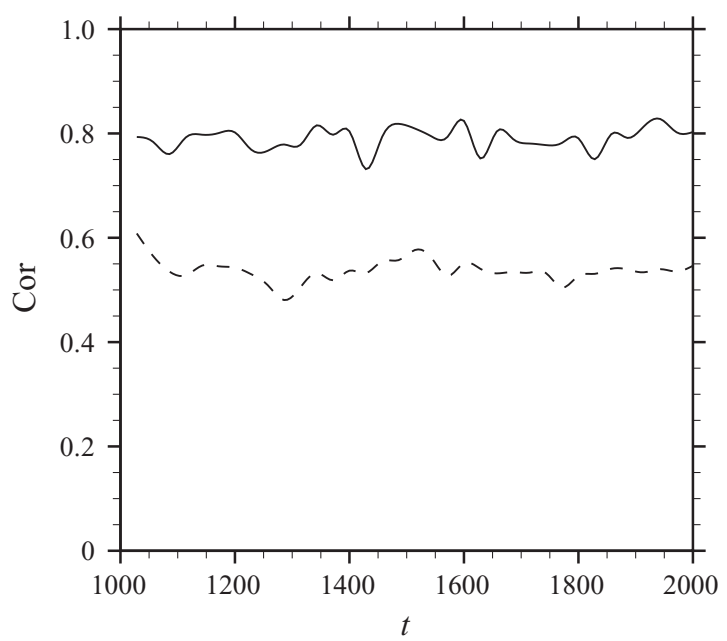

Figure 8. Correlation of surface age with $(a)$ surface temperature $(-)$ and $(b)$ surface gas flux (- ) during the simulation.

range in its neighbourhood the result of $\tau$ remains unchanged and because the physical meaning is clear.

We note that in (3.2), the surface temperature is used to quantify the Eulerian surface age $\tau_{T}$. We have also tested the use of surface gas flux $q_{g}$ to obtain the Eulerian surface age $\tau_{q_{g}}$ (for derivations see Appendix B),

$$
\tau_{q_{g}}=(\Delta c)^{2} \frac{D}{q_{g}^{2} \pi},
$$

in which the surface age is accordingly defined as $\tau=\tau_{L}+\tau_{q_{g}}$. We found that this approach for defining the surface age gives results not as good as the one using $\tau_{T}$ does. As shown in figure 7, $\tau$ changes as $C$ varies. As pointed out by Handler et al. (1999) and also as shown in $\S 3.1$, the surface gas flux is strongly affected by advection and hence it is more intermittent than the surface temperature field.

We also note that when the Eulerian surface age is quantified, vertical advection is not considered in the formulation (Appendix B). The advection effect is discussed in Appendix $\mathrm{C}$. The solution depends on the history of $\beta$ and is much more complex than the diffusion-only solution. The gas case is studied by Chan \& Scriven (1970) by using an analytical similarity solution, which results in (3.11) and (3.12), which are discussed in $\S 3.3$. For the heat case, similar analytical solution cannot be obtained and Appendix C presents numerical results only. Therefore, it is difficult to include the advection effect in quantifying the Eulerian surface age. However, it is found that if the surface temperature is used instead of the surface gas flux, the error caused by omitting advection is much smaller (Appendix C). We also remark that heat diffusion has been widely used in experiments to quantify surface age for gas transfer (see e.g. Jähne \& Haußecker 1998).

Figure 8 shows the correlation coefficient between surface age and surface temperature (Cor $\langle\tau, T\rangle$ ) and that between surface age and surface gas flux $\left(\operatorname{Cor}\left\langle\tau, q_{g}\right\rangle\right)$ during the non-dimensional simulation time $t=1000-2000$ (about 280 large-eddy turnover times), after the turbulence statistics have reached quasi-steady state. It is shown that $\operatorname{Cor}\langle\tau, T\rangle$ is larger than $\operatorname{Cor}\left\langle\tau, q_{g}\right\rangle$. As pointed out earlier, 

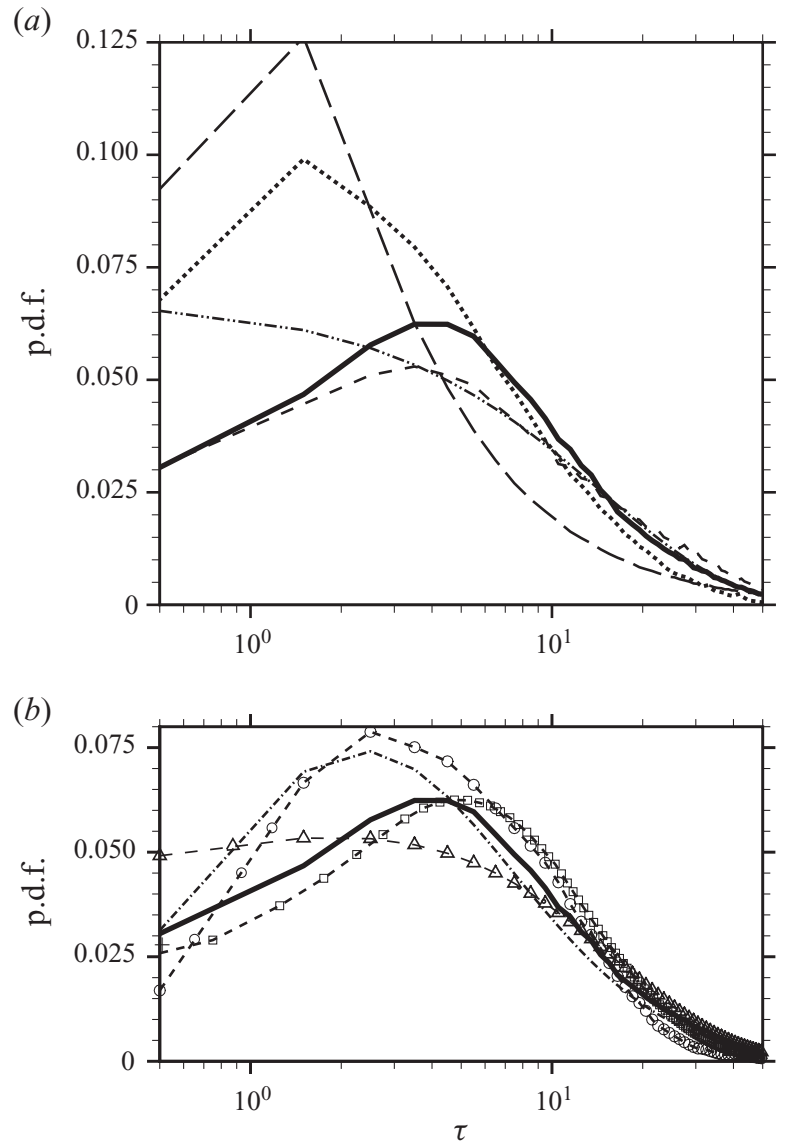

FIGURE 9. Probability density functions of surface age obtained from $(a)----$, solving the diffusion equation for temperature; _ — - solving the diffusion equation for gas; -..-, random surface renewal theory; $(b)-.-$, curve fitting with the lognormal distribution; $-\triangle-$, curve fitting with the gamma distribution; $-\square-$, modified surface renewal method; $-\bigcirc-$, corrected flux method. In $(a)$ and $(b)$, the thick solid lines represent the HLTT method.

compared with gas flux, temperature is less affected by downwellings and upwellings and thus serves as a more appropriate indicator of surface age.

\subsubsection{Probability density distribution of surface age}

Using the HLTT method, we obtain the surface age for each surface element. The resultant p.d.f. of surface age $\tau$ is plotted in figure $9(a, b)$ by the thick solid lines. At young surface age, as $\tau$ increases, the p.d.f. increases first, reaches a maximum around $\tau=5$, and then decreases.

In his random surface renewal theory, Danckwerts (1951) made two assumptions. The first is that all surface elements have an equal probability of being renewed by fresh fluid from the bulk flow, regardless of their surface age. Since the p.d.f. of surface age $\tau$, p.d.f. $(\tau)$, is governed by

$$
\frac{1}{\text { p.d.f. }(\tau)} \frac{\mathrm{d}(\text { p.d.f. }(\tau))}{\mathrm{d} \tau}=-s,
$$


where $s$ is the rate of renewal, which was assumed to be a constant in Danckwerts (1951), an exponential distribution of surface age can be derived:

$$
\text { p.d.f. }(\tau)=s \exp (-s \tau) \text {. }
$$

The second assumption in Danckwerts' theory is the pure diffusion of gas after surface renewal, which was originally suggested by Higbie (1935) and which gives the instantaneous gas flux as

$$
q_{g}(\tau)=\left(c_{t}-c_{b u l k}\right) \sqrt{\frac{D}{\pi \tau}} .
$$

Using (3.5) and (3.6), Danckwerts (1951) obtained the average surface gas flux as

$$
\left\langle q_{g}\right\rangle=\left(c_{t}-c_{b u l k}\right) \sqrt{D s} .
$$

Therefore, based on the numerical value of $\left\langle q_{g}\right\rangle$, one can estimate the value of $s$ as

$$
s=\frac{1}{D}\left(\frac{\left\langle q_{g}\right\rangle}{c_{t}-c_{\text {bulk }}}\right)^{2} .
$$

Figure $9(a)$ shows that the result of the HTLL method is substantially different from the exponential distribution (3.5) predicted by Danckwerts (1951) (using the $s$ value given by (3.8)), which decreases monotonically as $\tau$ increases. The issue of neglecting the advection effect in the gas transfer (the second assumption of Danckwerts 1951) has been discussed in the proceeding sections. Here, we discuss the rate of renewal $s$ (the first assumption of Danckwerts 1951). According to the kinematic boundary condition, the surface is material and no fluid particle can leave or join the surface. A fluid parcel from the bulk flow may very closely approach the surface, but it will not become a surface element, and the mechanism for the scalar transfer process is through molecular diffusion in the vicinity of the surface. A surface element cannot be replaced by surface renewal. It can only expand and shrink due to positive and negative surface divergence. The rate of change of its area $A$ is $\mathrm{d} A / \mathrm{d} \tau=A \mathrm{~d} u / \mathrm{d} x+A \mathrm{~d} v / \mathrm{d} y$, which can be written as

$$
\frac{1}{A} \frac{\mathrm{d} A}{\mathrm{~d} \tau}=\beta .
$$

The area percentage is identical to the p.d.f., which is governed by (3.4). Comparing (3.9) and (3.4), we can quantify $s$ as (Kermani \& Shen 2009)

$$
s=-\langle\beta\rangle .
$$

Figure 10 shows $s=-\langle\beta\rangle$ from our DNS data for different surface age. Contrary to Danckwerts' assumption, $s$ is not always a positive constant, but a function of surface age. Although $s$ approaches asymptotically to a constant positive value of around 0.07 at large $\tau$, it has large variations at small $\tau$. In particular, $s$ is negative when $\tau<5$, indicating that surface elements grow in area (positive surface divergence) at young surface age.

Figure $9(a)$ shows that for large surface age values, Danckwerts' theory agrees with the p.d.f. obtained from the HLTT method. This is consistent with the finding that $s$ is approximately constant at large $\tau$ as shown in figure 10. At young surface age, however, the exponential distribution is incorrect. In reality, surface elements in upwelling areas have young surface age. They expand for a limited time, and then start to shrink after they have left the upwelling areas. The assumption that $s$ has a constant positive value at all surface age is equivalent to assuming that all surface 
Average surface age

HLTT method

Pure diffusion method based on temperature

Pure diffusion method based on gas flux

Random surface renewal model

Corrected flux method

Modified surface renewal model
14.1

17.1

25.0

14.8

12.5

13.0

TABLE 1. Average surface age obtained from various methods.

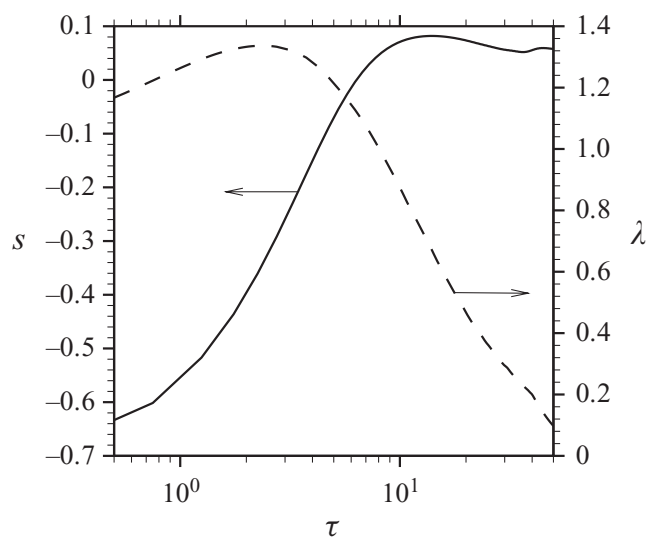

FIGURE 10. Variations of surface renewal rate $s(-)$ and the correction factor $\lambda$ for surface gas flux in (3.12) (--) with respect to surface age $\tau$.

elements shrink, which is not physically consistent. The stage of young surface age is when most of the interfacial transfer occurs (as discussed in more detail in $\$ 4.2$ ). Hence, it is crucial to accurately capture the surface age distribution for this stage.

\subsection{Comparison of the HLTT method with other methods}

In the HLTT method, the Lagrangian tracing part $\left(\tau_{L}\right)$ is defined according to the original physical meaning of the surface age. As discussed in $\S 3.2 .1$, the Eulerian part $\left(\tau_{T}\right)$ uses pure diffusion of heat that is an acceptable approximation for surface temperature. In addition, $\tau_{T}$ is much less than $\tau_{L}$ on average (figure 7 ). The probability distribution of $\tau_{T}$ is further illustrated in figure 11(a). As shown, except for small $\tau$ in which case surface particles enter upwelling regions relatively soon, the weight of $\tau_{T}$ is small. (For comparison, figure 11(b) shows that the result changes appreciably if $\tau_{T}$ is replaced by $\tau_{q_{g}}$; as pointed out in $\S 3.2 .1, \tau_{q_{g}}$ is affected by vertical advection and its diffusion-based solution should not be used to indicate surface age.) Therefore, we use the HLTT result as a base to discuss other methods for the quantification of surface age.

For comparison, we first consider methods based on the pure diffusion assumption, i.e. the Lagrangian tracing is not performed and the surface age is given by $\tau_{T}$ or $\tau_{q_{g}}$ directly. The obtained surface age p.d.f.s are shown in figure $9(a)$. We note that the p.d.f. obtained from heat pure diffusion is much closer to the HLTT result; on the other hand, there exist large differences between the gas pure diffusion and HLTT results. Table 1 lists the average values of surface age, of which the value from the HLTT method is 14.1. The average surface age obtained by the heat pure diffusion 

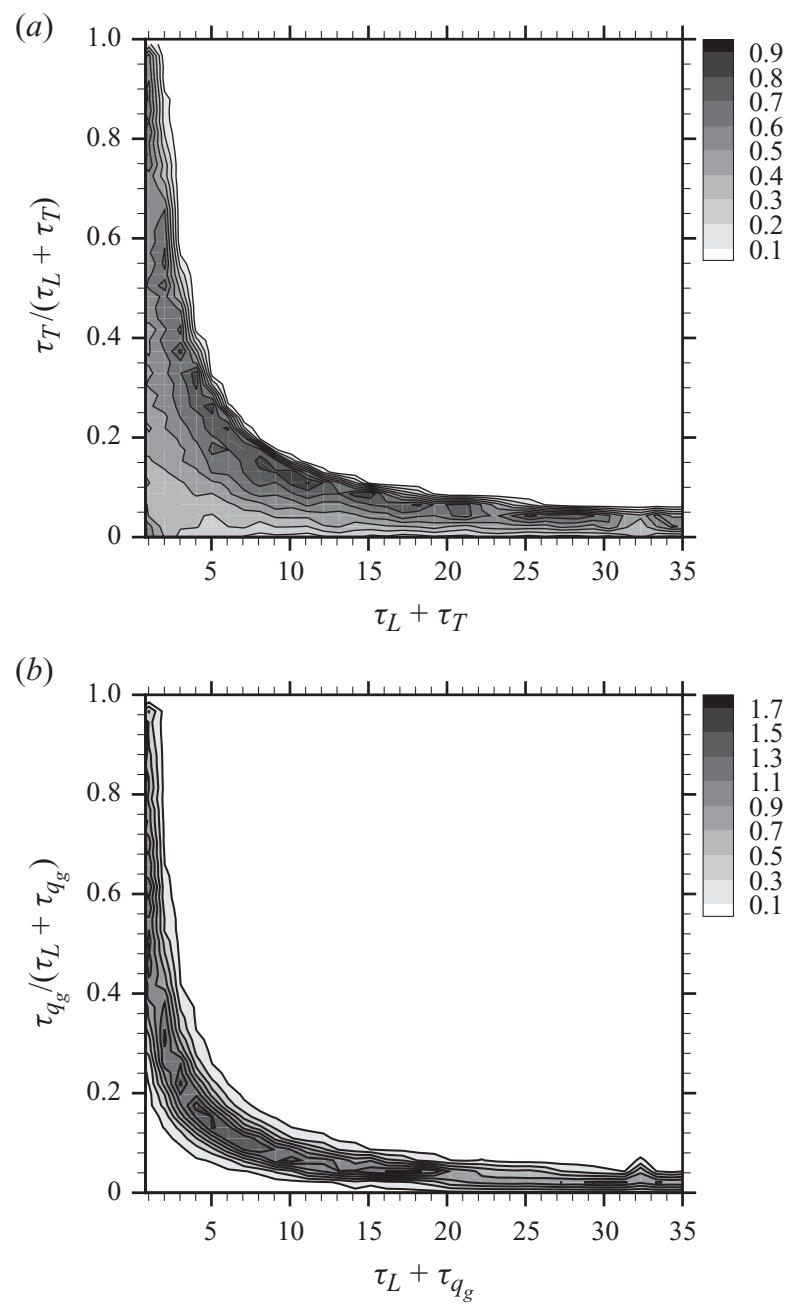

FIGURE 11. Propability distribution of the weight of the Eulerian part of the surface age in the total surface age obtained by the hybrid method: $(a) \tau_{T} /\left(\tau_{L}+\tau_{T}\right)$ and $(b) \tau_{q_{g}} /\left(\tau_{L}+\tau_{q_{g}}\right)$.

model is slightly larger. When the pure diffusion model based on surface gas flux is used, the deviation is large because gas flux is highly sensitive to vertical advection. We find that at old surface age, the downwelling reduces the gas flux so much that the resultant surface age is unrealistically high. A very significant portion of the surface area (about $19 \%$ ) has surface age even beyond the range plotted in figure $9(a)$. Therefore, the assumption of pure diffusion for gas is incorrect.

The average surface age from Danckwerts' random surface renewal model is surprisingly close to the HLTT result. As shown in $\S 3.2$, the exponential distribution works well for large values of surface age. At young surface age, however, the error in p.d.f. is significant. Therefore, Danckwerts' theory may provide a reasonably good estimate of the overall time scale of surface renewal. However, when detailed information is needed on the occurrence of the events for significant interfacial transfer, Danckwerts' theory should not be used. The main drawback of this theory is that it cannot distinguish and quantify interfacial transfer at different phases with respect to surface renewal (e.g. upwelling versus downwelling) and it does not consider the advection effect. 
Vertical advection (quantified by surface divergence) has been shown to play an essential role in interfacial gas transfer. To elaborate on this, we next discuss two auxiliary methods for surface age quantification. With the HLTT method, we obtain average surface divergence $\langle\beta\rangle$ as a function of surface age. The variation of $s=-\langle\beta\rangle$ with $\tau$ is shown in figure 10 . With such information, the gas diffusion method can be improved substantially if the advection effect is taken into account. In studying the upwelling stagnation free-surface flow, Chan \& Scriven (1970) modified the result of Danckwerts (1951) by considering the advection effect and rewrote the instantaneous gas transfer coefficient as

$$
K(\tau)=\lambda(\tau) \sqrt{\frac{D}{\pi \tau}} .
$$

Here $\sqrt{D / \pi \tau}=K_{0}(\tau)$ is the instantaneous gas transfer coefficient for the pure diffusion case, and the correction factor due to advection $\lambda(t)$ is

$$
\lambda(\tau)=2 \sqrt{D \tau} \frac{\exp \left[\int_{0}^{\tau} \beta\left(t^{\prime}\right) \mathrm{d} t^{\prime}\right]}{\left\{4 D \int_{0}^{\tau} \exp \left[2 \int_{0}^{t^{\prime}} \beta\left(t^{\prime \prime}\right) \mathrm{d} t^{\prime \prime}\right] \mathrm{d} t^{\prime}\right\}^{0.5}} .
$$

With (3.12) and the value of $\langle\beta\rangle(\tau)=-s(\tau)$ from the DNS results as shown in figure 10, we calculate the correction factor $\lambda(\tau)$ for gas flux, which is plotted in figure 10. It has unit value at $\tau=0$ (as given by (3.12)) and reaches its maximum value of 1.3 around $\tau=2.3$. After $\tau=2.3$, it decreases and returns to 1 around $\tau=8$, indicating that upwelling and downwelling effects cancel each other at that time. Afterwards, $\lambda$ continues to decrease because the downwelling effect at old surface age further reduces gas transfer.

On the basis of the $\lambda$ value obtained above and the instantaneous gas transfer coefficient $K(\tau)$ for each surface element, we calculate surface age $\tau$ using (3.11). We call this approach the corrected flux method. The distribution of surface age obtained from this method is shown in figure $9(b)$. Comparing this result with the distribution obtained from solving the pure diffusion equation for gas, we find that the new result is closer to the HLTT result, as expected. Table 1 shows that the value of average surface age is improved significantly.

We can also use the time variation of $s=-\langle\beta\rangle$ obtained from DNS to calculate the surface age p.d.f. by integrating (3.4). Using a Crank-Nicholson scheme, we obtain numerically the surface age p.d.f. as shown in figure $9(b)$. We call this approach the modified surface renewal method. The corresponding average surface age is listed in table 1, which is close to that of the HLTT method. The advantage of this modified surface renewal method over the traditional random surface renewal model is that the surface stretching and shrinking at different stages of surface renewal are captured by the variation of $s$ with $\tau$.

It is noted that both the corrected flux method and the modified surface renewal method rely on information of $\langle\beta\rangle(\tau)$, which is obtained from the HLTT method in our DNS study. Therefore, these two methods cannot be used independently, and the HLTT method still serves as the basis. The reason we discuss these two additional approaches is mainly to illustrate the importance of surface divergence.

Finally, we examine curve fitting of the surface age distribution obtained from the HLTT method, which is useful for parameterization in applications. Using likelihood analysis (Kass \& Raftery 1995) we compare the lognormal and gamma distributions 

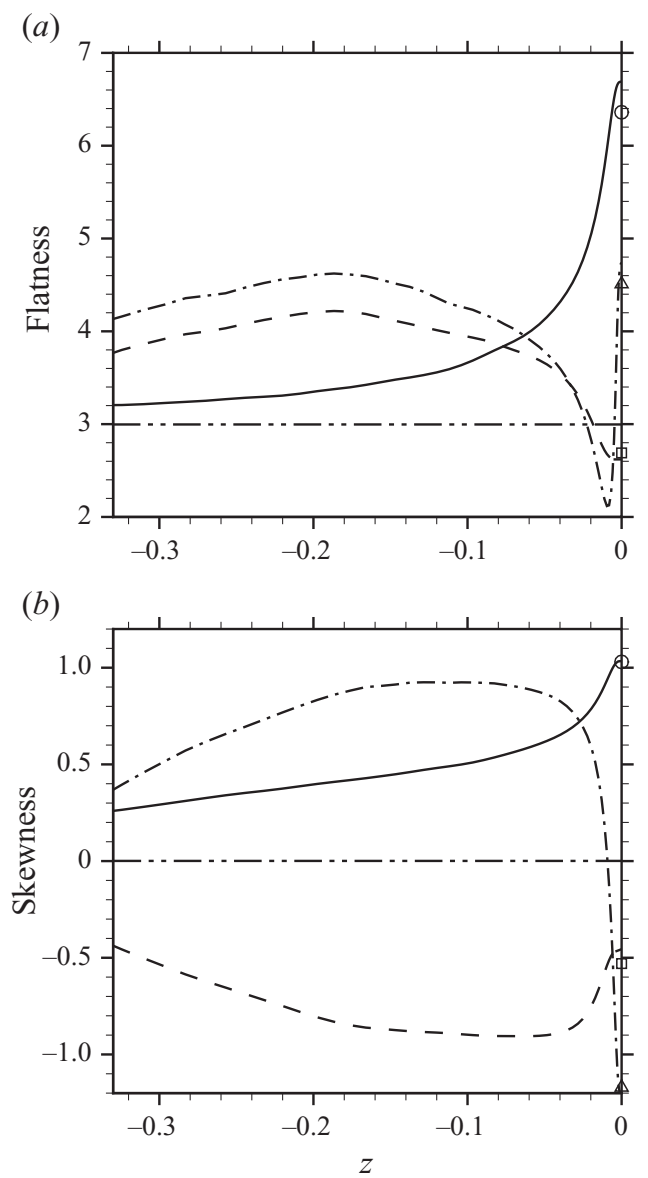

Figure 12. Profiles of $(a)$ flatness and $(b)$ skewness of $\longrightarrow$, vertical velocity $w$; - - , gas concentration $c$; -- , temperature $T ;-\cdots-$, Gaussian distribution. The symbols denote the results using the representative surface data obtained using the VISA windows.

shown in figure $9(b)$, and find that the former is slightly better. The curve fitting using the lognormal distribution is in agreement with the measurement results in the literature. The experiment by Rao, Narasimha \& Narayanan (1971) indicates that the time between bursts at a turbulent boundary layer has a lognormal distribution. Previous studies of open channel flows have shown that surface renewals are originated from bursts at the bottom boundary layer (see Komori et al. 1989; Rashidi et al. 1991). Therefore, the lognormal distribution can be used to describe surface renewal intermittency in open channel flows. For flows with deep water, measurements by Garbe et al. (2004) also show that the lognormal distribution provides a good approximation for surface age distribution.

\section{Scalar statistics}

\subsection{Statistical distribution of surface features}

\subsubsection{Probability density distribution}

Next, we investigate the statistics of flow and scalar quantities near the free surface. For the vertical velocity, temperature and gas concentration, figure $12(a, b)$ shows their 

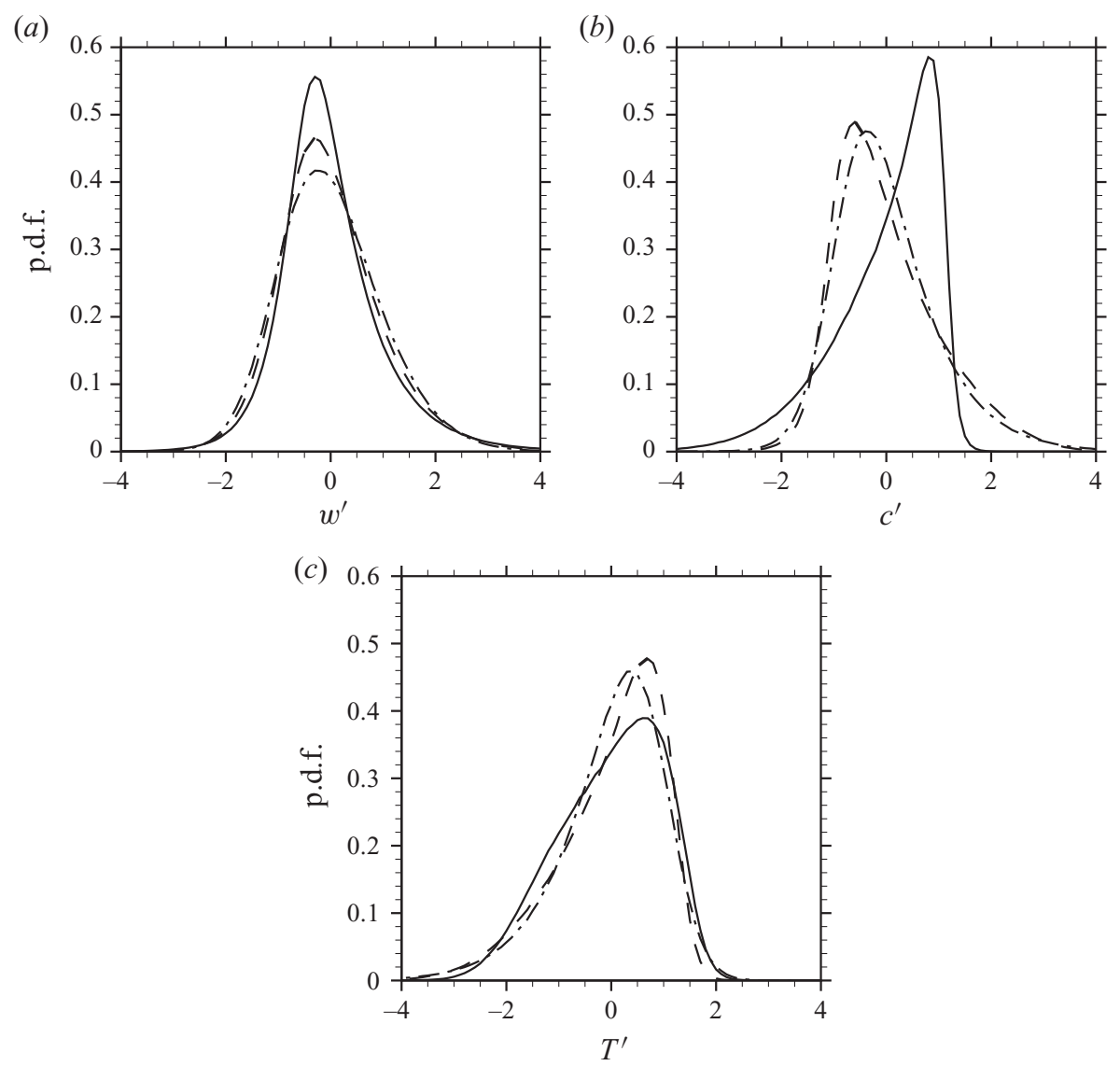

FIGURE 13. Probability density functions of fluctuations of $(a)$ vertical velocity $w^{\prime}$, $(b)$ gas concentration $c^{\prime}$ and $(c)$ temperature $T^{\prime}$ at various depths:,$- d_{t}^{+}=0.02 ;--, d_{t}^{+}=12$; $-\cdot-, d_{t}^{+}=42$. All variables are normalized by their r.m.s. values.

flatness and skewness profiles; figure $13(a-c)$ shows the p.d.f.s at three representative depths, $d_{t}^{+}=42,12$ and 0.02 . For the vertical velocity, as the free surface is approached, its flatness increases, with the tails of the p.d.f. becoming larger. Therefore, close to the free surface, relatively strong vertical motion is more frequent than in the bulk of the flow (Handler et al. 1999). The skewness of vertical velocity is positive and large close to the free surface, indicating that strong upwellings are more frequent than strong downwellings. This is related to the fact that fluid elements leaving upwelling areas will lose their kinetic energy due to dissipation before entering downwelling areas (Perot \& Moin 1995).

Upwelling brings fluid elements with low gas concentration towards the free surface. Therefore, negative $c^{\prime}$ is associated with upwelling. Very close to the free surface, the increase in flatness and the negative skewness (figures $12 a, b$ and $13 b$ ) are caused by the stronger effect of upwelling compared to that of downwelling on gas transport. Figures $12(b)$ and $13(b)$ show that as the free surface is approached, the skewness reverses sign, with the dominant tail of the p.d.f. switching from the right to the left. This behaviour of the p.d.f. of $c^{\prime}$ will be explained in $\S 4.1 .3$ in terms of the statistics of upwelling patterns. 
Downwelling has a strong effect on heat transfer. Some strong downwellings are located close to strong upwellings (see e.g. figure $5 a$ ). These downwellings absorb surface elements that have recently been pushed away from nearby upwelling areas, which have high temperature. However, downwellings that are not close to strong upwellings absorb surface elements that have been on the surface for a long time. In the latter case, the surface elements have been under outgoing flux of heat for a long period and are cold. This explains why figures $12(b)$ and $13(c)$ show a negative skewness with a large left tail in the p.d.f. of $T^{\prime}$. Figure 12(a) shows that near the free surface, the flatness of $T^{\prime}$ is closer to the Gaussian distribution compared with that of gas. The smaller flatness of temperature compared with that of gas concentration indicates that the temperature has less extreme fluctuations. This is consistent with the results shown in $\S 3$.

\subsubsection{Patterns of surface features}

The flatness, skewness and p.d.f.s shown in figures 12 and 13 are useful summaries about the properties of the vertical velocity, gas concentration and temperature. However, they are only point statistics, i.e. they are derived from these quantities observed one point at a time, collected without regard to the underlying spatial relation. Hence, these statistical quantities do not directly provide information about the structure of the flow. Two-point statistics such as auto-correlation functions can be used to obtain some limited information about this structure. However, in our visual observations of the turbulent flow field, particularly at the surface, we have noted well-defined spatial patterns that can be associated with significant upwelling events. Hence, in our investigation, we have chosen to characterize the surface field by analysing the upwelling patterns using spatial statistics, rather than two-point statistics. It is shown below how this statistical analysis can be applied to provide insights into the underlying processes in the flow field.

We begin with the variable-interval space averaging (VISA) method (see e.g. Kim 1983; Piomelli, Yu \& Adrian 1996; Shen et al. 2004) to obtain a representative data set from the surface flow field. In our VISA procedure, we detect upwelling events by searching local peaks of surface divergence. The $(x, y)$ coordinates are then shifted so that the peaks are centred at the origin. Then the surface divergence values $\beta(x, y)$ in a $43 \times 23$ array of pixels (located on grid points) are collected along with the corresponding VISA windows for the temperature fluctuation $T^{\prime}(x, y)$ and gas flux fluctuation $q_{g}^{\prime}(x, y)$. Here, the window size and thus the pixel number are determined based on the turbulence velocity integral scale. A series of 1238 windows capturing the significant upwelling events were selected in this manner. This data set is representative in that the one-point statistics computed from this series of windows are nearly identical with those for the entire surface field. This is indicated in figure 12, where the flatness and skewness based on the data within these windows (shown with symbols) are compared with the corresponding statistics for the entire field. The reason why the windowed data set is representative is that the window size is sufficiently large to contain the upwelling pattern as well as the surrounding turbulent flow field. Another way to interpret this data collection procedure is to note that a sampling of $43 \times 23$ pixel windows randomly placed in the flow field would be an alternative method for obtaining a data set that is statistically representative of the entire field. Each window would almost always contain upwelling events, and the VISA method simply shifts these patterns to the centre. The centring procedure in VISA is important because it allows further quantitative analysis of the spatial patterns associated with the upwellings. 

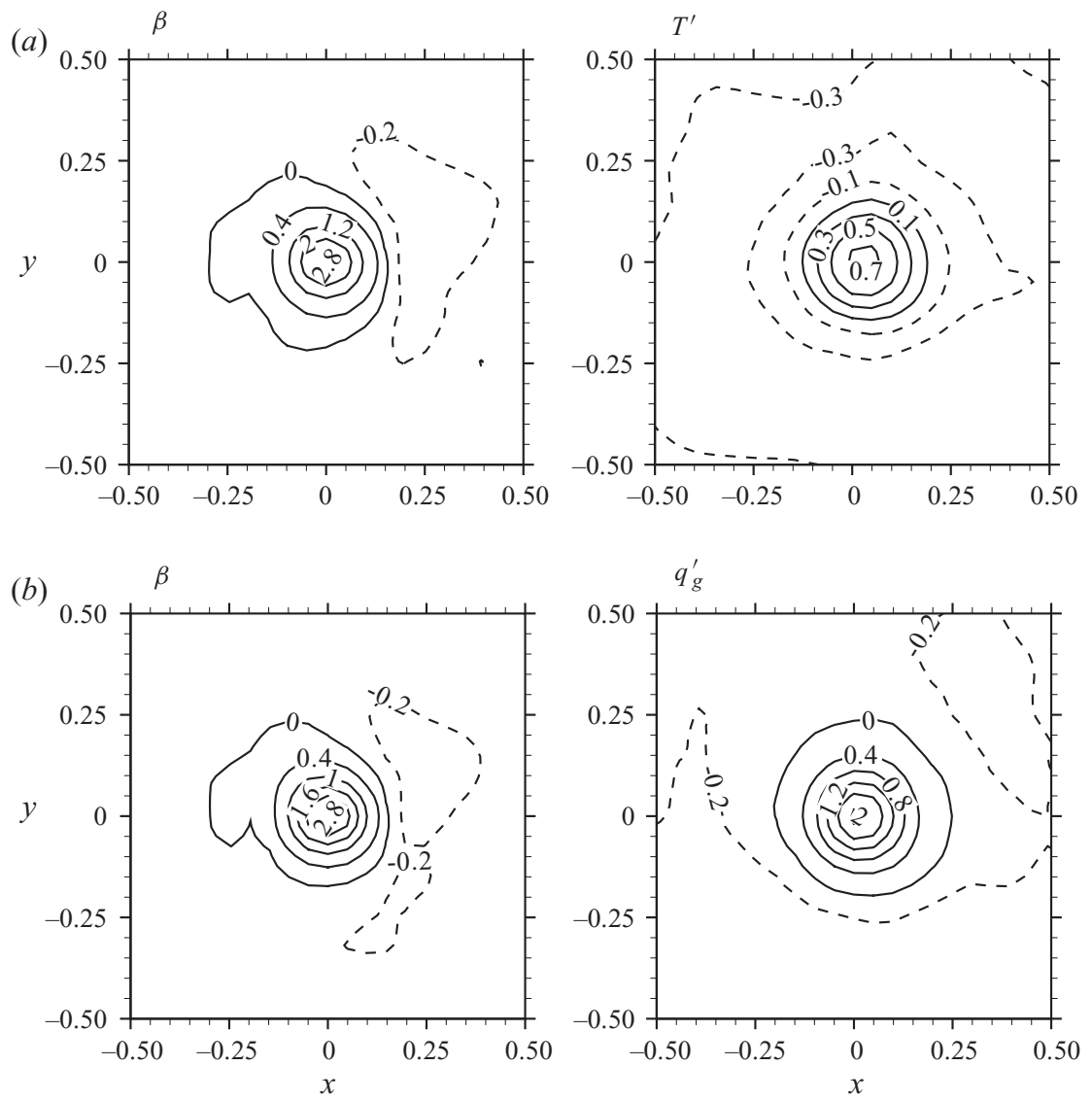

FIGURE 14. Representative patterns of (a) surface divergence $\beta$ and temperature fluctuation $T^{\prime}$, (b) surface divergence $\beta$ and gas flux fluctuation $q_{g}^{\prime}$, as obtained by statistical clustering. Dashed contour lines represent negative values.

The surface fields are very irregular, but with proper statistical analysis it is possible to identify the dominant patterns associated with the upwelling. Simple averaging is inappropriate because multiple patterns are associated with each field. In this paper, a clustering procedure based on the expectation-maximization algorithm (Hastie, Tibshirani \& Friedman 2001) is used. To capture the relationships between $\beta$ and $T^{\prime}$ and between $\beta$ and $q_{g}^{\prime}$, each VISA window of surface divergence data is appended with the corresponding windows of temperature or gas flux data. The patterns identified by clustering have a surprisingly smooth structure. Example patterns for surface-field pairs are shown in figure 14(a,b). These patterns can be approximated by two-dimensional Gaussian surfaces, with functional form

$$
f\left(r ; a, b, r_{0}\right)=a \exp \left(-\frac{r^{2}}{2 r_{0}^{2}}\right)+b,
$$

where $r=\sqrt{x^{2}+y^{2}}$ is the radial coordinate. The parameter $r_{0}$ is the length scale associated with the width of the peak, which is a measure of the turbulence macroscale of the corresponding quantity $\left(\beta, T^{\prime}\right.$, or $\left.q_{g}^{\prime}\right)$. For distances $r$ significantly distant from the centre $\left(r / r_{0}>2\right)$, the surface approaches the far-field value $b$. The amplitude 


$\begin{array}{lccc} & \beta & T^{\prime} & q_{g}^{\prime} \\ \text { Mean of the amplitude, } a & 3.84 & 1.43 & 2.93 \\ \text { Mean of the length scale, } r_{0} & 0.070 & 0.148 & 0.102 \\ \text { Mean of the far-field value, } b & -0.078 & -0.064 & -0.087 \\ \text { R.m.s of the random field, } \sigma_{e} & 0.91 & 0.81 & 0.85\end{array}$

TABLE 2. Amplitude and length-scale parameters of the two-dimensional Gaussian models for the observed patterns of surface divergence $\beta$, surface temperature fluctuation $T^{\prime}$ and surface gas flux fluctuation $q_{g}^{\prime}$. Here, $\beta, T^{\prime}$ and $q_{g}^{\prime}$ are normalized by their surface r.m.s. values.

of the peak value is $a$. The actual fields of the surface divergence, temperature or gas flux are not as smooth as the Gaussian surface in (4.1) and an additive random field is needed to capture the spatial variability with length scales smaller than $r_{0}$. If we denote the surface divergence, temperature or gas flux by the generic field variable $\xi(x, y)$, then the statistical model for $\xi$ would be given by

$$
\xi(x, y)=a \exp \left(-\frac{x^{2}+y^{2}}{2 r_{0}^{2}}\right)+b+e(x, y),
$$

where $e(x, y)$ is a zero-mean random field. Since the downwellings are considerably weaker and less structured than the upwellings, they are included in $e(x, y)$.

While clustering is used to identify the functional form for the surface field patterns, nonlinear regression is needed to obtain the numerical estimates for the parameters. The results for the mean values of $a, b$ and $r_{0}$ as well as the r.m.s. $\sigma_{e}$ of the random field $e(x, y)$ are shown in table 2.

It is found that the far-field values for $b$ can be modelled as constants for the surface divergence and gas flux. For the temperature, however, it varies considerably about the mean value of -0.064 . It is also found that there is a linear relationship between the amplitude and the corresponding far-field value such that the sum is nearly constant, given by the sum of mean values, $a+b \approx 1.37$. This is physically consistent because $a+b$ is the maximum temperature at the peak of the upwelling, and this temperature is bounded by the temperature in the flow underneath.

If the length-scale parameters $r_{0}$ for the three field quantities are compared, it can be seen that the length scales for the temperature and gas flux are about $100 \%$ and $50 \%$ larger than that for the surface divergence, respectively. This significant difference in length scales can be observed in the single realization of the flow field illustrated in figure 6, where the temperature and gas flux have peaks that are broader than the divergence. The results in table 2 , however, are based on a statistical analysis of all of the observed upwelling events selected by the VISA method, and are more conclusive than individual observations such as shown in figure 6 .

\subsubsection{Discussion}

It was noted in the beginning of $\S 4.1 .2$ that the VISA-generated windowed data set is representative of the surface flow field. Hence, we use the spatial description of the flow and scalar patterns in the preceding subsection as a basis for a qualitative discussion of the one-point statistics, particularly the marginal p.d.f.s shown in figure 15(a).

We begin with a short derivation of the p.d.f. associated with the Gaussian shape of the upwelling, as given by (4.1). The cumulative distribution function (c.d.f.) of $\xi$ for the fundamental case where there is no additive random field (so that $e(x, y)=0$ 


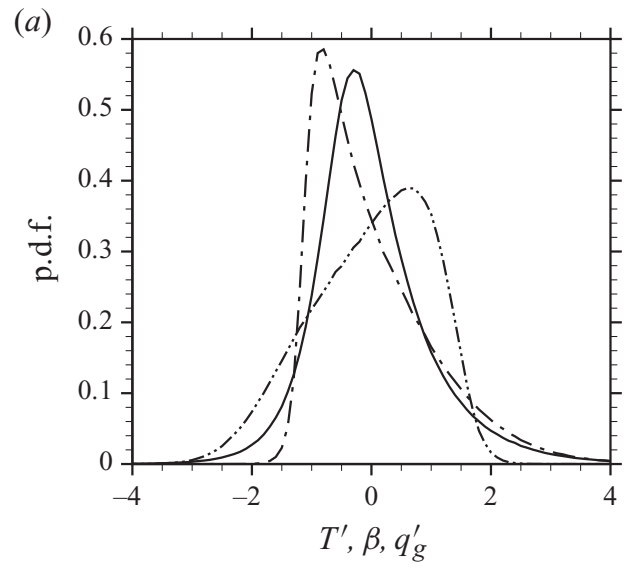

(b)

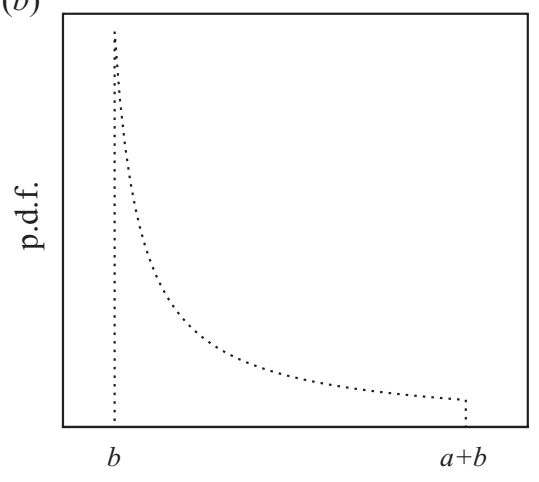

(c)

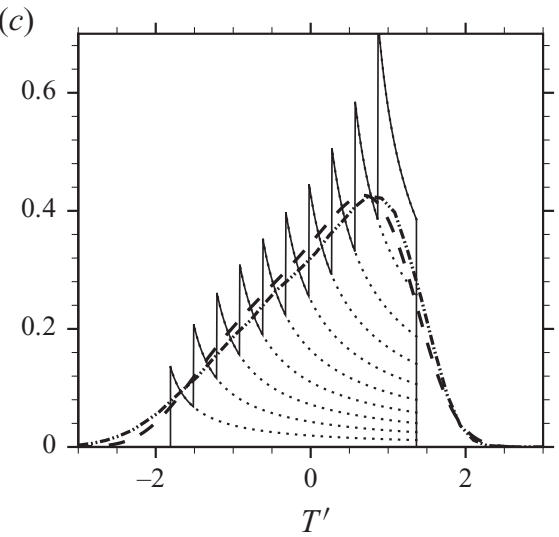

FIGURE 15. (a) DNS results for the p.d.f.s of surface characteristics: $\beta ;-\cdots-$, surface temperature fluctuation $T^{\prime} ;-\cdot-$, surface gas flux fluctuation $q_{g}^{\prime}$. (b) $\cdots$, schematics illustrating the model results for upwelling p.d.f. given by (4.6). (c) $\cdots$, upwelling p.d.f.s of $T^{\prime}$ given by (4.6) with different $b$ values; - - superposition of upwelling p.d.f.s for $T^{\prime} ;--$, smoothed superposed p.d.f. of $T^{\prime} ;-\cdots-$, p.d.f. of $T^{\prime}$ obtained by DNS. All variables are normalized by their r.m.s. values.

in (4.2)) is, by definition

$$
\text { c.d.f. }(\xi)=P\left[a \exp \left(-\frac{x^{2}+y^{2}}{2 r_{0}^{2}}\right)+b \leqslant \xi\right],
$$

where $b<\xi_{0} \leqslant \xi \leqslant a+b$ in which the lower limit $\xi_{0}$ is determined shortly and $P$ stands for probability. To derive an analytical expression for the probability, we use polar coordinates to obtain

$$
\text { c.d.f. }(\xi)=P\left[a \exp \left(-\frac{r^{2}}{2 r_{0}^{2}}\right) \leqslant \xi-b\right]=P\left[r \geqslant r_{1}\right]=1-P\left[r<r_{1}\right],
$$

where $r_{1}=r_{0} \sqrt{2 \log (a /(\xi-b))}$. The c.d.f. can be written in terms of the area $A$ of the window:

$$
\text { c.d.f. }(\xi)=1-\frac{\pi r_{1}^{2}}{A}=1-\frac{2 \pi r_{0}^{2}}{A} \log \frac{a}{\xi-b} .
$$


From this we obtain the lower limit $\xi_{0}$ for $\xi$ by solving c.d.f. $\left(\xi_{0}\right)=0$ or $\left(2 \pi r_{0}^{2} / A\right) \log \left(a /\left(\xi_{0}-b\right)\right)=1$ with solution $\xi_{0}=b+a \exp \left(-A /\left(2 \pi r_{0}^{2}\right)\right)$. The p.d.f. is then obtained by differentiation

$$
\text { p.d.f. }\left(\xi ; a, b, r_{0}\right)=\frac{\mathrm{d}(\text { c.d.f. }(\xi))}{\mathrm{d} \xi}=-\frac{2 \pi r_{0}^{2}}{A} \frac{\mathrm{d}}{\mathrm{d} \xi}\left(\log \frac{a}{\xi-b}\right)=\frac{2 \pi r_{0}^{2}}{A} \frac{1}{\xi-b} \propto \frac{1}{\xi-b} \text {. }
$$

This p.d.f., illustrated in figure $15(b)$, has a peak at the left corresponding to the far-field value $b$ and a tail towards the right that ends at the peak amplitude $a+b$.

In the statistical model in (4.2), a zero-mean random field $e(x, y)$ is added to the surface upwelling patterns. This field has probability density p.d.f. (e) with standard deviations listed in table 2. Based on an analysis of the DNS data, it was found that the p.d.f. of this random field is nearly symmetric for the surface divergence and temperature and is positively skewed for the gas flux. The p.d.f. for the sum of the upwelling patterns and zero-mean random field is given by the convolution

$$
\text { p.d.f. }(\xi)=\int \text { p.d.f. }(\xi-e) \text { p.d.f. }(e) \text { de }
$$

This convolution is essentially a filtering operation which would smooth and broaden the original p.d.f.

The parameters $a, b$ and $r_{0}$ describing the upwelling pattern shapes are also random variables. The effect of the randomness of these parameters on the p.d.f. of $\xi$ can be represented by a second convolution

$$
\text { p.d.f. }(\xi)=\iiint \text { p.d.f. }\left(\xi ; a, b, r_{0}\right) \text { p.d.f. }\left(a, b, r_{0}\right) \mathrm{d} a \mathrm{~d} b \mathrm{~d} r_{0},
$$

which, in general, must be evaluated numerically. The expression in (4.6)-(4.8) along with the information in table 2 comprises the statistical model for the surface fields. In the following, it is shown how this model can be used to interpret the marginal and joint p.d.f.s of the surface fields. We begin with the marginal p.d.f.s, shown in figure 15(a), and in the next subsection we discuss the joint p.d.f.s.

For the divergence and gas flux, the net effect of the two successive convolutions in (4.7) and (4.8) is to smooth the fundamental p.d.f. of the surface upwelling in (4.6). This can be observed by comparing the relatively smooth p.d.f.s for the divergence and gas flux in figure $15(a)$ with the underlying p.d.f. of the surface upwelling shown in figure $15(b)$. Since the random field $e(x, y)$ associated with the gas flux is positively skewed, as noted above, the p.d.f. of the gas flux has a higher positive skew than the p.d.f. of the divergence. The p.d.f. for the temperature field has a triangular shape with a negative rather than a positive skew. In the following, we use the convolution in (4.8) to explain how this unusual shape would arise given that the underlying p.d.f. of the upwelling is positively skewed as shown in figure $15(b)$.

To visualize this convolution, we approximate the multiple integral in (4.8) as a weighted sum and display the result as a superposition of p.d.f.s. This is shown by the dotted lines in figure 15(c), where each of the p.d.f.s corresponds to a p.d.f. $\left(\xi ; a, b, r_{0}\right)$ in (4.6) with a different set of parameters. The most significant variability is in the parameter $b$, which varies approximately from -1.80 to 0.87 ; as noted earlier the sum $a+b$ is approximately 1.37. Hence, as shown in figure $15(c)$, the superposition of upwelling p.d.f.s has a constant upper bound, $a+b$, and a varying lower bound, $b$. This causes an accumulation of probability density at the upper bound and a nearly linear slope towards the left. 


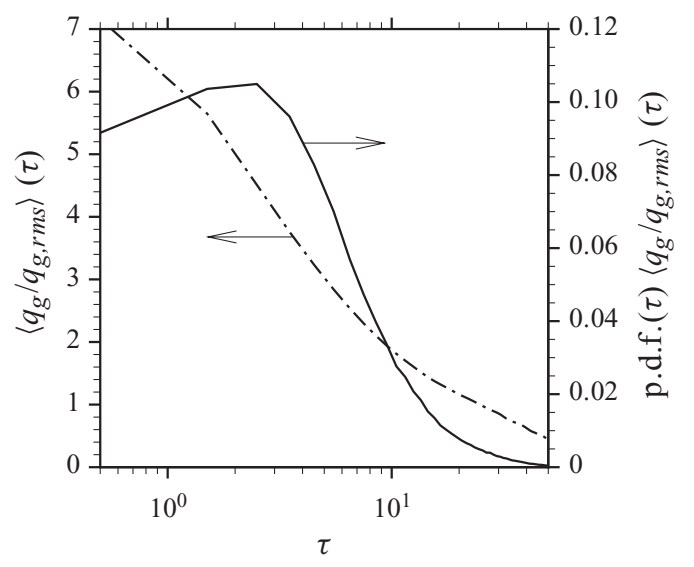

Figure 16. Average surface gas flux $(-\cdot-)$ and p.d.f. of surface gas flux $(-)$ as functions of surface age. The surface age is obtained by the HLTT method.

The p.d.f. of the zero-mean fluctuations $e(x, y)$ is nearly symmetric and has only a minor smoothing effect on the final p.d.f. for the temperature field. The final approximate representation of the statistical model in (4.6)-(4.8), given by a smoothing of superposed p.d.f.s, is shown by the dashed curve in figure 15(c), which closely follows the negatively skewed p.d.f. of the surface temperature obtained by DNS shown by the dash-double dotted line.

Finally, we note that the positive and negative skewness in the p.d.f.s for the gas concentration $c$ at $d_{t}^{+}=12$ and 0.02 in figure $13(b)$ can be explained in a similar manner to that for the positive and negative skewness in the gas flux and temperature p.d.f.s. For most of the fluid, the skewness would be positive, in the same direction as the fundamental upwelling p.d.f. in figure $15(b)$. Very close to the free surface at $d_{t}^{+}=0.02$, however, the concentration $c$ is bounded above, as was the case for temperature. Hence, the superposition and convolution of upwelling p.d.f.s for $c$ are similar to the curves shown in figure $15(c)$, leading to the negative skewness shown in figure $13(b)$.

\subsection{Scalar statistics with respect to surface age}

The availability of surface age information makes it possible to investigate the statistics of scalar transport with respect to different stages of surface renewal, from which a better understanding of interfacial scalar transfer can be obtained. In this section, we first illustrate the dependence of scalar statistics on surface age. We then present the joint p.d.f.s of surface divergence with surface temperature and gas flux.

Figure 16 shows the variation of the average normalized surface gas flux $\left\langle q_{g} / q_{g, r m s}\right\rangle$ with the surface age $\tau$. In calculating the statistics, $q_{g}$ is obtained directly from DNS at each surface element. The surface age $\tau$ of each surface element is quantified using the HTLL method as discussed earlier. The results for $q_{g} / q_{g, r m s}$ are then averaged, conditioned upon each $\tau$, to yield the curve of $\left\langle q_{g} / q_{g, r m s}\right\rangle(\tau)$ shown in figure 16. Also plotted is the product of p.d.f. $(\tau)$ and $\left\langle q_{g} / q_{g, r m s}\right\rangle(\tau)$, which denotes the contribution to the interfacial gas transfer per unit surface age. It can be seen that surface elements with young surface age have large gas flux. This is due to two reasons: the small thickness of the gas surface layer that increases molecular diffusion and the upward advection associated with upwelling. Despite the high flux, surface elements with very young surface age do not have the maximum contribution to interfacial gas 


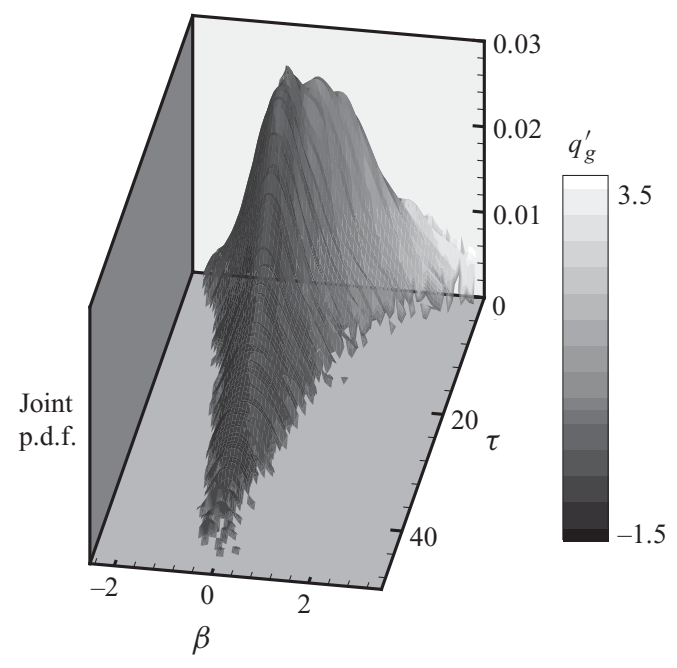

FIGURE 17. Three-dimensional joint p.d.f. of surface age $\tau$, surface divergence $\beta$ and surface gas flux fluctuation $q_{g}^{\prime}$. Here $\beta$ and $q_{g}^{\prime}$ are normalized by their r.m.s. values.

transfer because they cover only a small fraction of the surface area, as reflected by the relatively small value of p.d.f. $(\tau)$ (see figure 9). As the surface age increases, p.d.f. $(\tau)$ increases while the average gas flux decreases. The combined effects result in a maximum contribution around $\tau=3$. As the surface age further increases, the average gas flux continues to decrease, because the gas surface layer becomes thicker and because the advection turns downwards due to downwelling; the p.d.f. $(\tau)$ also begins to decrease with $\tau$ (figure 9). As a result, surface elements of relatively old surface age have less contribution to interfacial gas transfer, as shown in figure 16.

Figure 17 provides a comprehensive picture on the relations between surface age, surface divergence and surface gas flux. In the figure, the contour surface represents the joint p.d.f. of surface age and divergence and the colour is the mean surface gas flux. As pointed out earlier, upwellings have young surface age, and strong downwellings may also have small values of surface age because they are often located close to strong upwellings. Therefore, a wide joint p.d.f. at small $\tau$ is shown in figure 17. Figure 17 also shows that high $q_{g}^{\prime}$ occurs in the high $\beta$-small $\tau$ region, while low $q_{g}^{\prime}$ occurs in regions of large negative $\beta$ or large $\tau$, consistent with the results discussed earlier.

Figure 18( $a, b)$ shows the joint p.d.f.s of surface divergence with surface temperature and surface gas flux. It can be seen that the upper portion of these p.d.f.s exhibits a very distinct rightward shift of the probability density. In the following, this is explained qualitatively in terms of the physical behaviour of the flow. Then a quantitative analysis is presented in terms of the statistical model of upwellings in $\S 4.1$.

Qualitatively, upwelling brings up warm fluid elements from the bulk flow, so that large positive $T^{\prime}$ is associated with large positive surface divergence $\beta$. As mentioned earlier, some strong downwellings are near strong upwellings, and absorb warm surface elements from them. Therefore, large negative $\beta$ is also associated with positive $T^{\prime}$. Cold surface regions (negative $T^{\prime}$ ) are usually associated with fluid elements that have left upwelling for a long time. Most of them have not arrived at the downwelling regions yet, and thus have small $\beta$ (but at the end they are absorbed by downwellings). Their temperature is quite low due to the long duration 

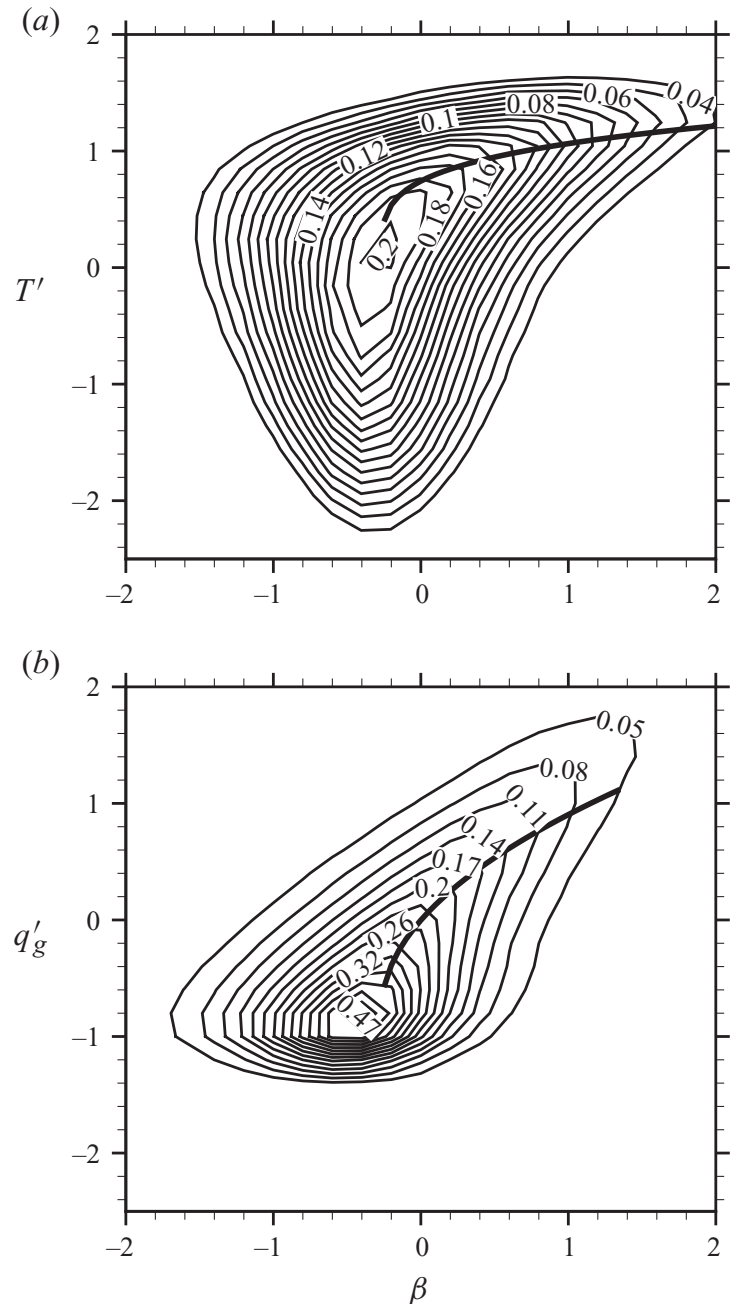

FIGURE 18. Joint probability density functions of $(a)$ surface divergence $\beta$ and surface temperature fluctuation $T^{\prime}$, and $(b)$ surface divergence $\beta$ and surface gas flux fluctuation $q_{g}^{\prime}$. All variables are normalized by their r.m.s. values. The thick lines are peaks of the p.d.f.s of $T^{\prime}$ and $q_{g}^{\prime}$ conditioned on $\beta$ as predicted by the model of (4.9).

of outgoing heat flux. Figure 18(a,b) indicates that surface divergence has a more sharply defined statistical relationship with gas flux than with temperature. As shown earlier, unlike surface temperature, surface gas flux is affected immediately by surface divergence. This result is in agreement with the study of Lakehal et al. $(2008 a)$ and Lakehal et al. (2008b), who showed that the quadrant II $\left(u^{\prime}<0\right.$ and $\left.w^{\prime}>0\right)$ has the largest contribution to the Reynolds stress and interfacial transfer among different quadrants.

To gain further insight into the characteristics of the joint p.d.f.s, we first briefly describe the shape of these p.d.f.s that would result if the surface fields were assumed to be independent. The resulting joint p.d.f. would be given by the product of the empirical marginal p.d.f.s of the zero-mean error fields. For instance, the hypothetical joint p.d.f. for the surface divergence and temperature under the independence 
assumption would be p.d.f. $\left(\beta, T^{\prime}\right)=$ p.d.f. $(\beta)$ p.d.f. $\left(T^{\prime}\right)$. These hypothetical p.d.f.s would look very similar to the actual joint p.d.f.s in figure 18 at the lower portions of these plots. The upper portions, however, would be considerably different. The actual joint p.d.f.s have a distinct rightward shift at higher values of $T^{\prime}$ and $q_{g}^{\prime}$. This implies that the fields are dependent, which is expected from the discussion of the relationships between upwellings and the surface temperature and gas flux in $\S \S 3.1$ and 4.1 .

We can study the dependence relationships more closely by examining the conditional mode, which is the peak of the joint p.d.f. for a fixed value of one of the random variables. For instance, in figure 18(a), the conditional mode of the surface divergence $\beta$ can be found by finding the peak value of the joint p.d.f. along a horizontal line corresponding to a fixed value of the temperature $T^{\prime}$. The conditional modes can be visualized by the ridge of the joint p.d.f. For $T^{\prime}<0$, the conditional mode is nearly constant at approximately $\beta=-0.4$, but veers sharply to higher value as $T^{\prime}$ increases above 0 . Similarly, on examining figure $17(b)$, it can be seen that the conditional mode of $\beta$ is at approximately -0.4 for gas flux $q_{g}^{\prime}<-1$ and veers to higher values as $q_{g}^{\prime}$ increases above -1 . In other words, the conditional modes show that high values for the temperature and gas flux are associated with positive surface divergence.

We conclude this section by showing the relationships between the statistical model of upwellings in $\S 4.1$ and the behaviour of the joint p.d.f.s in figure 17(a,b). Specifically, we presented expressions in $\S 4.2$ to derive a predictive analytical model for the conditional mode that closely matches the observational results shown in these figures. We begin with the pair $\beta$ and $T^{\prime}$ and the corresponding surfaces defined by (4.2). Then we simply eliminate the spatial variable $r$. We perform a similar derivation to obtain the relation between $\beta$ and $q_{g}^{\prime}$. The results for the conditional modes are

$$
T^{\prime}=b_{T}+a_{T}\left(\frac{\beta-\breve{b}_{\beta}}{a_{\beta}}\right)^{c}, \quad q_{g}^{\prime}=\breve{b}_{q_{g}}+a_{q_{g}}\left(\frac{\beta-\breve{b}_{\beta}}{a_{\beta}}\right)^{d}
$$

where $c=r_{0, \beta}^{2} / r_{0, T}^{2}, d=r_{0, \beta}^{2} / r_{0, q_{g}}^{2}$ and where $\breve{b}_{\beta}=-0.27, \breve{b}_{q_{g}}=-0.8$ are the modes of the surface divergence and gas flux, obtained by finding the maximum of the marginal p.d.f.s in figure 15 .

We substitute the parameter values from table 2 into (4.9) and plot the resulting curves over the joint p.d.f.s to obtain our model predictions for the conditional modes. The figure shows a close match between the predictions from the statistical model and the actual shape of the joint p.d.f.s obtained directly from the DNS-generated data. Further insight can be obtained by taking the derivatives of (4.9) with respect to $\beta$ to get the slopes of the conditional modes. It can be seen that these slopes are proportional to the exponents $c$ and $d$, which are inversely proportional to $r_{0, T}^{2}$ and $r_{0, q_{g}}^{2}$, respectively. From table 2, it can be seen that $r_{0, T}^{2}$ is over twice as large as $r_{0, q_{g}}^{2}$, so that the slope of the conditional mode for $T^{\prime}$ should be less than half of the slope of the conditional mode for $q_{g}^{\prime}$. This is confirmed by the shapes of the joint p.d.f.s in figure 18(a,b). This shows how the statistical model of the upwelling shapes in $\S 4.1$ can be used to quantify the relationships between surface divergence, temperature and gas flux. 


\section{Conclusions}

In this study, we have investigated transport of passive scalars near a free surface with a focus on surface renewal statistics. When an upwelling occurs, fresh fluid from the bulk flow is brought towards the surface. As a result, the vertical gradient of gas concentration is increased and interfacial gas transfer is enhanced. In the case of surface cooling, the surface temperature is increased after the upwelling brings warm fluid from below.

The DNS performed in this study provides detailed information on the complex structure of upwellings. Upwellings can occur individually or in interacting groups. Upwellings can also be affected by other surface events such as surface-connected vortices in the neighbourhood. Around an upwelling, downwellings often exist. After a fluid particle emanates from an upwelling, it may enter a nearby downwelling immediately, or it may travel on the surface for some time before it is absorbed by a downwelling. Results show that gas flux is strongly dependent on and responds rapidly to the upwellings and downwellings. The surface temperature, on the other hand, responds only gradually to the vertical advection.

To quantify surface age for the description of the different stages of surface renewal, we implement an HLTT method, based on which flow and scalar statistics at different stages of surface renewal are investigated. The results show that the rate of surface renewal $s$ cannot be assumed to be constant, so that modifications are needed in the random surface renewal model (Danckwerts 1951). At young surface age, surface elements are located at upwelling regions and the areas of the elements increase due to surface stretching; only afterwards does the surface divergence, i.e. $-s$, become negative and asymptotically approach a constant value. The crucial stage of young surface age, when most of interfacial gas transfer occurs, is not accounted for in the model of Danckwerts (1951). The current study addresses this by using time integration based on the $s(t)$ value. Knowledge of $s(t)$ also leads to a corrected flux model that considers both advection and diffusion in gas transfer. Our study also shows that in some studies, if pure diffusion has to be assumed due to the lack of advection information, heat diffusion provides acceptable results at young surface age.

To obtain a statistical description of the upwelling, we performed a clustering analysis using a method based on the expectation-maximization algorithm. While the surface fields are very irregular, it is found that the dominant two-dimensional surface patterns associated with the upwellings have a Gaussian shape. Using this Gaussian functional form for the upwelling patterns, nonlinear regression is used to obtain the numerical estimates for the parameters for the surface divergence, gas flux and temperature. It is then shown how this statistical description of the upwelling can be used to explain the skewness in the p.d.f.s, including the reason for the opposite directions of skewness in the temperature and gas flux fields. The influence of the upwelling on these surface quantities is also analysed through a quantitative description of the asymmetry in the surface divergence-temperature and surface divergence-gas flux joint p.d.f.s. The results demonstrate how the slower diffusion time associated with temperature as compared with gas transfer affects the surface distribution p.d.f.s.

Finally, we remark that this study serves as a first step in our investigation of gas and heat transfer at the water surface; we intentionally kept the problem set-up simple to allow a detailed mechanistic study of some of the fundamental physics. The assumption that temperature and gas have, respectively, Neumann and Dirichlet 
free-surface boundary conditions is based on the ratio of air-side and water-side resistances of interfacial transfer; this approach has been used successfully in other DNS studies in the literature. For a further understanding of the problem, it would be desirable to consider air and water as a coupled system in the simulation (cf. Lin et al. 2008; Lakehal et al. 2008a; Liu et al. 2009; Komori et al. 2010). It would be helpful to evaluate the surface age in the air and water coupled shear flow. In future studies, it would also be desirable to consider other aspects of the problem such as the effects of surfactants, waves and wind-wave interactions on the statistics of surface renewal.

We would like to thank the referees for their valuable comments that provided us significant help to improve the previous version of this paper.

\section{Appendix A. Discussion on the case with zero gas flux at the bottom}

In our simulation, the gas concentration at the bottom boundary is assumed to be constant. However, in many laboratory experiments (e.g. those using tanks), there is no gas flux at the bottom; the time variation of the concentration of the dissolved gas is often used to quantify the gas flux at the surface. In this appendix, we show that the different bottom boundary conditions for the scalar do not affect the essentials of the near-surface scalar transport, which is the focus of the present study.

We have performed a separate simulation with zero gas flux at the bottom boundary. Meanwhile, the gas concentration is fixed at the free surface and gas flux exists there. As time increases, the dissolved gas field becomes more uniform to approach the surface value. It is noted that the governing advection-diffusion equation for the passive scalar (2.3) is linear with respect to $c$. After the turbulence scalar field has fully developed, we normalize $c$ by the difference in the concentrations at the bottom and top boundaries. It is found that the normalized scalar statistics vary little at different times (not shown here due to space limitation).

We next normalize $c$ by the friction gas concentration (defined in $\S 2.3$ ) at the free surface, and compare its statistics with those discussed in $\S 2.3$. Figure 19 shows the results of the mean- and fluctuation-intensity profiles. The difference in the near-bottom region is as expected, because the Neumann and Dirichlet boundary conditions are used respectively at the bottom. However, for the near-surface region that the present study focuses on, these two cases are indiscernible, indicating that the physics of the near-surface scalar transport is the same, even with different bottom conditions.

\section{Appendix B. Quantification of surface age based on pure diffusion of gas or heat}

We summarize in this appendix the methods for the quantification of surface age based on the pure diffusion assumption, which were widely used in the literature. Considering diffusion in the predominant vertical direction only, the governing equation for the scalar concentration $f$ is

$$
\frac{\partial f}{\partial \tau}=D \frac{\partial^{2} f}{\partial z^{2}} .
$$

In the gas case, $f=c$, and its solution subject to the Dirichlet boundary condition $\left(c=c_{t}\right.$ at $z=0$ and $c=c_{\text {bulk }}$ at $\left.z=-\infty\right)$ and uniform initial condition $\left(c=c_{\text {bulk }}\right.$ at $\tau=0$ for $z<0$; i.e. the scalar field below the surface is assumed to be perfectly mixed 

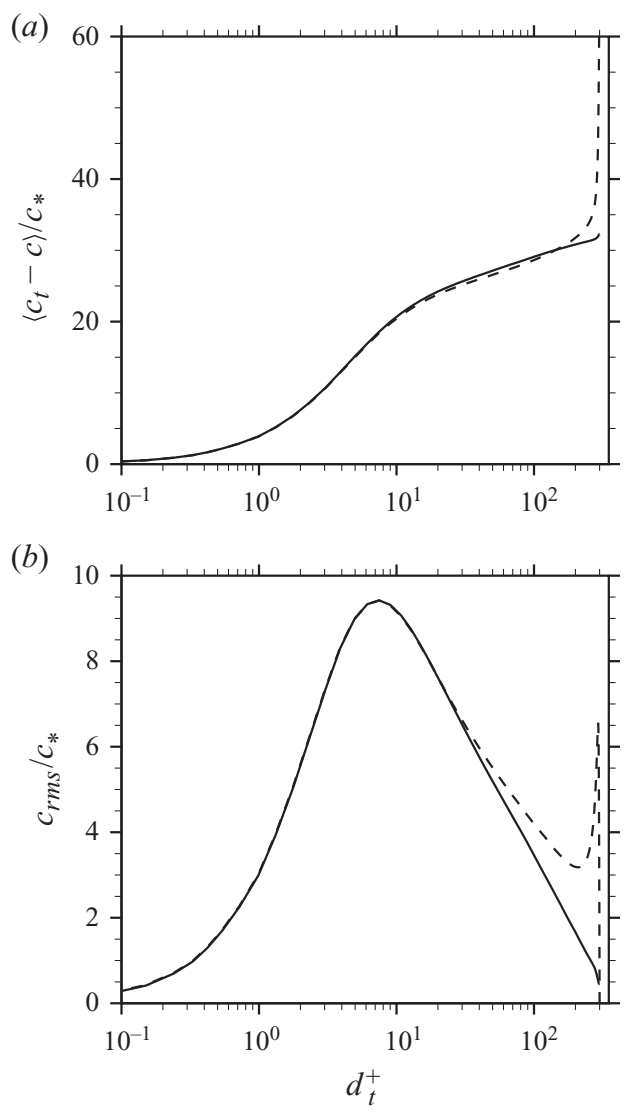

FIGURE 19. Normalized profiles of $(a)$ gas concentration $\left\langle c_{t}-c\right\rangle / c_{*}$ and $(b)$ r.m.s. of gas concentration fluctuations $c_{r m s} / c_{*}$ near the free surface: - - gas with the Neumann bottom boundary condition; - - , gas with the Dirichlet bottom boundary condition. Note that the mean gas concentration is shifted to have zero value at the free surface.

after the surface renewal; see Higbie 1935) is

$$
\frac{c-c_{\text {bulk }}}{c_{t}-c_{\text {bulk }}}=\operatorname{erfc}(-\xi)
$$

Here $\xi=z / 2 \sqrt{(D \tau)}$ is the normalized distance from the surface and erfc is the complementary error function. The derivative of (B 2) with respect to $z$ results in

$$
D \frac{\partial c}{\partial z}=\left(c_{t}-c_{b u l k}\right) \sqrt{\frac{D}{\pi \tau}} \exp \left(-\xi^{2}\right) .
$$

For a fluid particle residing at the surface $(\xi=0)$, its surface age is found accordingly,

$$
\tau=\left(c_{t}-c_{b u l k}\right)^{2} \frac{D}{q_{g}^{2} \pi} \text {. }
$$

Since the instantaneous surface gas flux $q_{g}$ is known for all surface elements in our DNS, we can quantify the surface age for each element using (B 4).

In the heat case, we define $f=T_{\text {bulk }}-T(z, \tau)$ in (B 1). Neumann boundary condition applies to $f$ at the free surface, $D \partial f / \partial z=-D \partial T / \partial z=-q_{h}$, where $q_{h}$ is the heat flux at the surface. With the aforementioned uniform initial condition, the solution is 


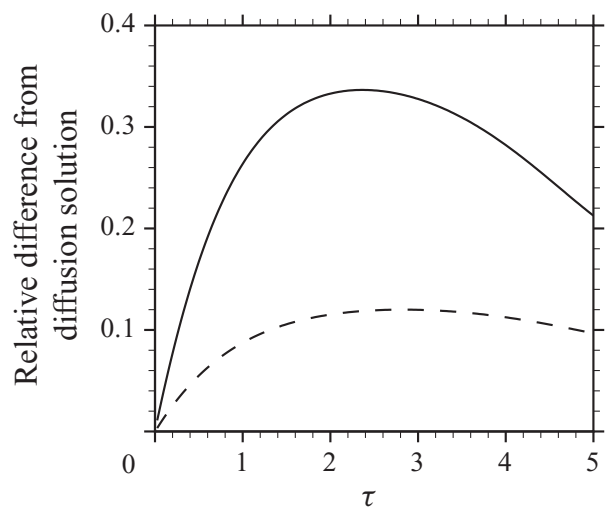

FIGURE 20. Relative differences between the solutions of the advection-diffusion equation and the pure diffusion equation for: - - , surface flux in the gas case; --, surface temperature in the heat case.

(Soloviev \& Schlussel 1996)

$$
T_{\text {bulk }}-T(z, \tau)=-2 q_{h}\left(\sqrt{\frac{\tau}{D}}\left(\frac{\exp \left(-\xi^{2}\right)}{\sqrt{\pi}}+\xi \operatorname{erfc}(-\xi)\right)\right) .
$$

In DNS, since the temperature of all surface elements $T_{t}$ is known, (B 5) with $\xi=0$ can be used to find the surface age,

$$
\tau=\left(\frac{T_{b u l k}-T_{t}}{2 q_{h}}\right)^{2} \pi D .
$$

\section{Appendix C. Effect of vertical advection on scalar transfer}

To add the advection effect to the cases discussed in Appendix B, we consider the advection-diffusion equation

$$
\frac{\partial f}{\partial \tau}-\beta z \frac{\partial f}{\partial z}=D \frac{\partial^{2} f}{\partial z^{2}},
$$

which has an analytical solution for the gas case (Chan \& Scriven 1970) but not the heat case. To examine the characteristics for both gas and heat, we use a second-order Crank-Nicholson scheme to solve (C1) numerically. The value of $\beta(\tau)$ is described by a fourth-order polynomial fit of the average surface divergence of our DNS result (figure 10):

$$
\beta(\tau)=0.003 \tau^{4}-0.041 \tau^{3}+0.2182 \tau^{2}-0.6101 \tau+0.8372, \quad \tau \leqslant 5 .
$$

Details of the solution are provided in Kermani (2010). Here we discuss only the difference in the final results between (B 1) and (C1). We normalize the difference by the solution of (B 1) and plot the relative difference in figure 20. Compared with the pure diffusion result, in the case of heat, the surface temperature is increased by upwelling because fluid elements with high temperature are brought from the bulk flow towards the free surface. In the case of gas, upwelling results in larger gas concentration gradient near the free surface and thus larger gas flux. When $\beta$ has the variation described by (C2), as $\tau$ increases, the relative error first increases and then decreases as the strength of the upwelling decreases. The relative difference in surface 
temperature is smaller than that of gas flux. (Using a perturbation solution, Kermani \& Shen 2009 argued that the leading-order errors are three times different.) Therefore, if the pure-diffusion equation is used instead of the advection-diffusion equation in the quantification of surface age, the temperature-based result has a smaller error compared with the gas flux-based result. Hence, in our hybrid method for surface age quantification, we choose the surface temperature to be combined with Lagrangian tracing as discussed in $\S 3.2$.

\section{REFERENCES}

Atmane, M. A. \& George, J. 2002 Gas transfer across a zero-shear surface: a local approach. In Gas Transfer at Water Surfaces (ed. M. A. Donelan, W. M. Drennan, E. S. Saltzman \& R. Wanninkhof), pp. 255-259. American Geophysical Union.

BAnerJeE, S. 1990 Turbulence structure and transport mechanisms at interfaces. In Proc. Ninth Intl Heat Transfer Conf., pp. 395-418. Hemisphere.

Banerjee, S., Lakehal, D. \& Fulgosi, M. 2004 Surface divergence models for scalar exchange between turbulent streams. Intl J. Multiphase Flow 30, 963-977.

BANERJEe, S., Scott, D. S. \& Rhodes, E. 1968 Mass transfer to falling wavy liquid films in turbulent flow. Indust. Engng Chem. Fund. 7, 22-27.

Borue, V., Orszag, S. A. \& Staroselsky, I. 1995 Interaction of surface waves with turbulence: direct numerical simulations of turbulent open-channel flow. J. Fluid Mech. 286, 1-23.

Brumley, B. H. \& JiRKa, G. H. 1988 Air-water transfer of slightly soluble gases: turbulence, interfacial processes and conceptual models. J. Phys.-Chem. Hydrodyn. 10, 295-319.

Campbell, J. A. \& Hanratty, T. J. 1982 Mass transfer between a turbulent fluid and a solid boundary: Linear theory. AIChE J. 28, 988-993.

Campbell, J. A. \& Hanratty, T. J. 1983 Mechanism of turbulent mass transfer at a solid boundary. AIChE J. 29, 221-229.

Chan, W. C. \& Scriven, L. E. 1970 Absorption into irrotational stagnation flow. Indust. Engng Chem. Fund. 9, 114-120.

Chu, C. R. \& Jirka, G. H. 1992 Turbulent gas flux measurements below the air-water interface of a grid-stirred tank. Intl J. Heat Mass Transfer 35, 1957-1968.

DANCKWERTS, P. V. 1951 Significance of liquid-film coefficients in gas absorption. Indust. Engng Chem. 43, 1460-1467.

Fortescue, G. E. \& Pearson, J. R. A. 1967 On gas absorption into a turbulent liquid. Chem. Engng Sci. 22, 1163-1176.

Garbe, C. S., Schimpf, U. \& Jähne, B. 2004 A surface renewal model to analyze infrared image sequences of the ocean surface for the study of air-sea heat and gas exchange. J. Geophys. Res. 109, C08S15.

Handler, R. A., Saylor, J. R., Leighton, R. I. \& Rovelstad, A. L. 1999 Transport of a passive scalar at a shear-free boundary in fully developed turbulent open channel flow. Phys. Fluids 11, 2607-2625.

Handler, R. A., Swean, T. F., Leighton, R. I. \& Swearingen, J. D. 1993 Length scales and the energy balance for turbulence near a free surface. AIAA J. 31, 1998-2007.

Harriott, P. 1962 A random eddy modification of the penetration theory. Chem. Engng Sci. 17, 149-154.

Hasegawa, Y. \& Kasagi, N. 2008 Systematic analysis of high Schmidt number turbulent mass transfer across clean, contaminated and solid interfaces. Intl J. Heat Fluid Flow 29, 765-773.

HasegaWA, Y. \& Kasagi, N. 2009 Hybrid DNS/LES of high Schmidt number mass transfer across turbulent air-water interface. Intl J. Heat Mass Transfer 52, 1012-1022.

Hastie, T., Tibshirani, R. \& Friedman, J. 2001 The Elements of Statistical Learning: Data Mining, Inference, and Prediction. Springer.

Herlina \& Jirka, G. H. 2008 Experiments on gas transfer at the air-water interface induced by oscillating grid turbulence. J. Fluid Mech. 594, 183-208.

Higbie, R. 1935 The rate of absorption of a pure gas into a still liquid during short periods of exposure. Trans. AIChE 31, 365-389. 
Hunt, J. C. R. \& Graham, J. M. R. 1978 Free-stream turbulence near plane boundaries. J. Fluid Mech. 84, 209-235.

Jähne, B. \& Haußecker, H. 1998 Air-water gas exchange. Annu. Rev. Fluid. Mech. 30, 443-468.

Johnk, R. E. \& Hanratty, T. J. 1962 Temperature profiles for turbulent flow of air in a pipe. I. The fully developed heat transfer. Chem. Engng Sci. 17, 867-879.

KADER, B. A. 1981 Temperature and concentration profiles in fully turbulent boundary layers. Intl J. Heat Mass Transfer 24, 1541-1544.

Kasagi, N., Tomita, Y. \& Kuroda, A. 1992 Direct numerical simulation of passive scalar field in a turbulent channel flow. J. Heat Transfer 114, 598-606.

Kass, R. E. \& Raftery, A. E. 1995 Bayes factors. J. Am. Stat. Assoc. 90, 773-795.

Kermani, A. 2010 Numerical study of turbulence and scalar transport process in free-surface and multi-phase flows. PhD thesis, Johns Hopkins University, Maryland.

Kermani, A. \& Shen, L. 2009 Surface age of surface renewal in turbulent interfacial transport. Geophys. Res. Lett. 36, L10605.

Khakpour, H. R., Shen, L. \& Yue, D. K. P. 2011 Transport of passive scalar in turbulent shear flow under a clean or surfactant-contaminated free surface. J. Fluid Mech. 670, 527-557.

KIM, J. 1983 On the structure of wall-bounded turbulent flows. Phys. Fluids 26, 2088-2097.

KIM, J. 1988 Investigation of heat and momentum transport in turbulent flows via numerical simulations. In Transport Phenomena in Turbulent Flows: Theory, Experiment, and Numerical Simulation (ed. M. Hirata \& N. Kasagi), pp. 715-729. Hemisphere.

KIM, J. \& MorN, P. 1985 Application of a fractional-step method to incompressible Navier-Stokes equations. J. Comput. Phys. 59, 308-323.

Komori, S., Kurose, R., Iwano, K., Ukai, T. \& Suzuki, N. 2010 Direct numerical simulation of wind-driven turbulence and scalar transfer at sheared gas-liquid interfaces. J. Turbul. 11 (32), $1-20$.

Komori, S., Murakami, Y. \& Ueda, H. 1989 The relationship between surface-renewal and bursting motions in an open-channel flow. J. Fluid Mech. 203, 103-123.

Komori, S., Nagaosa, R., Murakami, Y., Chiba, S., Ishit, K. \& Kuwahara, K. 1993 Direct numerical simulation of three-dimensional open-channel flow with zero-shear gas-liquid interface. Phys. Fluids A 5, 115-125.

Lakehal, D., Fulgosi, M., Banerjee, S. \& Yadigaroglu, G. $2008 a$ Turbulence and heat exchange in condensing vapor-liquid flow. Phys. Fluids 20, 065101.

Lakehal, D., Fulgosi, M. \& Yadigaroglu, G. 2008b Direct numerical simulation of condensing stratified flow. J. Heat Transfer 130, 021501.

Lakehal, D., Fulgosi, M., Yadigaroglu, G. \& Banerjee, S. 2003 Direct numerical simulation of turbulent heat transfer across a mobile, sheared gas-liquid interface. J. Heat Transfer 125, $1129-1139$.

Lamont, J. C. \& ScotT, D. S. 1970 An eddy cell model of mass transfer into the surface of turbulent liquid. AIChE J. 16, 513-519.

Lin, M., Moeng, C., Tsai, W., Sullivan, P. P. \& Belcher, S. E. 2008 Direct numerical simulation of wind-wave generation processes. J. Fluid Mech. 616, 1-30.

Liss, P. S. \& Slater, P. G. 1974 Flux of gases across the air-sea interface. Nature 247, 181-184.

Liu, S., Kermani, A., Shen, L. \& Yue, D. K. P. 2009 Investigation of coupled air-water turbulent boundary layers using direct numerical simulations. Phys. Fluids 21, 062108.

Lyons, S. L., Hanratty, T. J. \& Mclaughlin, J. B. 1991 Direct numerical simulation of passive heat transfer in a turbulent channel flow. Intl J. Heat Mass Transfer 34, 1149-1161.

Magnaudet, J. \& Calmet, I. 2006 Turbulent mass transfer through a flat shear-free surface. J. Fluid Mech. 553, 155-185.

McCready, M. J. \& Hanratty, T. J. 1984 A comparison of turbulent mass transfer at gas-liquid and solid-liquid interfaces. In Gas Transfer at Water Surfaces (ed. W. Brutsaert \& G. H. Jirka), pp. 283-292. Reidel.

McCready, M. J., Vassiliadou, E. \& Hanratty, T. J. 1986 Computer simulation of turbulent mass transfer at a mobile interface. AIChE J. 32, 1108-1115.

McKenna, S. P. \& McGillis, W. R. 2004 The role of free-surface turbulence and surfactants in air-water gas transfer. Intl J. Heat Mass Transfer 47, 539-553.

Moin, P. \& Mahesh, K. 1998 Direct numerical simulation: A tool in turbulence research. Annu. Rev. Fluid. Mech. 30, 539-578. 
MÜNSTERER, T. \& J̈̈HNE, B. 1998 LIF measurements of concentration profiles in the aqueous mass boundary layer. Exp. Fluids 25, 190-196.

Na, Y. \& HanRatTy, T. J. 2000 Limiting behavior of turbulent scalar transport close to a wall. Intl J. Heat Mass Transfer 43, 1794-1758.

Na, Y., Papavassiliou, D. V. \& Hanratty, T. J. 1999 Use of direct numerical simulation to study the effect of Prandtl number on temperature fields. Intl J. Heat Fluid Flow 20, 187-195.

Nagaosa, R. \& Handler, R. A. 2003 Statistical analysis of coherent vortices near a free surface in a fully developed turbulence. Phys. Fluids 15, 375-394.

Nakajima, M., Fukui, K., Ueda, H. \& Mizushina, T. 1980 Buoyancy effects on turbulent transport in combined free and forced convection between vertical parallel plates. Intl J. Heat Mass Transfer 23, 1325-1336.

Pan, Y. \& BANERJEe, S. 1995 A numerical study of free-surface turbulence in channel flow. Phys. Fluids 7, 1649-1664.

Perot, B. \& Moin, P. 1995 Shear-free turbulent boundary layers. Part 1. Physical insight into near-wall turbulence. J. Fluid Mech. 295, 199-227.

Piomelli, U., Yu, Y. \& AdRian, R. J. 1996 Subgrid-scale energy transfer and near-wall turbulence structure. Phys. Fluids 8, 215-224.

RaO, K. N., Narasimha, R. \& Narayanan, M. A. B. 1971 The 'bursting' phenomenon in a turbulent boundary layer. J. Fluid Mech. 48, 339-352.

Rashidi, M., Hetsroni, G. \& BanerJee, S. 1991 Mechanisms of heat and mass transport at gas-liquid interfaces. Intl J. Heat Mass Transfer 34, 1799-1810.

SchwertFirm, F. \& Manhart, M. 2007 DNS of passive scalar transport in turbulent channel flow at high Schmidt numbers. Intl J. Heat Fluid Flow 28, 1204-1214.

Shen, L., Triantafyllou, G. S. \& Yue, D. K. P. 2001 Mixing of a passive scalar near a free surface. Phys. Fluids 13, 913-926.

Shen, L., Yue, D. K. P. \& Triantafyllou, G. S. 2004 Effect of surfactants on free-surface turbulent flows. J. Fluid Mech. 506, 79-115.

Soloviev, A. V. \& Schlussel, P. 1996 Evolution of cool skin and direct air-sea gas transfer coefficient during daytime. Boundary-Layer Meteorol. 77, 45-68.

Sugihara, Y. \& Tsumori, H. 2005 Surface-renewal eddies at the air-water interface in oscillatinggrid turbulence. In Environmental Hydraulics and Sustainable Water Management (ed. J. H. W. Lee \& K. M. Lam), pp. 199-205. Taylor and Francis.

Tamburrino, A., Aravena, C. \& Gulliver, J. S. 2007 Visualization of 2-D divergence on the free surface and its relation to gas transfer. In Transport at the Air-Sea Interface (ed. C. S. Garbe, R. A. Handler \& B. Jähne), pp. 73-86. Springer.

TAmburrino, A. \& Gulliver, J. S. 2002 Free-surface turbulence and mass transfer in a channel flow. AIChE J. 48, 2732-2743.

Turney, D. E., Smith, W. C. \& BanerJee, S. 2005 A measure of near-surface fluid motions that predicts air-water gas transfer in a wide range of conditions. Geophys. Res. Lett. 32, L04607.

Zappa, C. J., Asher, W. E., Jessup, A. T., Klinke, J. \& Long, S. R. 2004 Microbreaking and the enhancement of air-water transfer velocity. J. Geophys. Res. 109, C08S16.

Zhang, C., Shen, L. \& Yue, D. K. P. 1999 The mechanism of vortex connection at a free surface. J. Fluid Mech. 384, 207-241.

Zhu, Y. \& Antonia, R. A. 1993 Temperature dissipation measurements in a fully developed turbulent channel flow. Exp. Fluids 15, 191-199. 